\title{
Two distinct trophectoderm lineage stem cells from human pluripotent stem cells
}

Adam Mischler ${ }^{1}$, Victoria Karakis ${ }^{1}$, Jessica Mahinthakumar ${ }^{1}$, Celeste Carberry ${ }^{2}$, Adriana San Miguel $^{1}$, Julia Rager ${ }^{2}$, Rebecca Fry², and Balaji M. Rao ${ }^{1,3^{*}}$

1Department of Chemical and Biomolecular Engineering, North Carolina State University, Raleigh, North Carolina 27695, USA

${ }^{2}$ Department of Environmental Sciences and Engineering, Institute for Environmental Health Solutions, University of North Carolina - Chapel Hill, Chapel Hill, North Carolina 27599, USA

${ }^{3}$ Golden LEAF Biomanufacturing Training and Education Center (BTEC), North Carolina State University, Raleigh, North Carolina 27695, USA

${ }^{*}$ Corresponding author: Email: bmrao@ncsu.edu 


\section{Summary}

2 Trophoblasts are the principal cell type of the placenta. The use of human trophoblast stem cells

3 (hTSCs) as a model for studies of early placental development is hampered by limited genetic

4 diversity of existing hTSC lines, and constraints on using human fetal tissue or embryos needed

5 to generate additional cell lines. Here we report the derivation of two distinct stem cells of the

6 trophectoderm lineage from human pluripotent stem cells. The first is a CDX2- stem cell equiva-

7 lent to primary hTSCs - they both exhibit identical expression of key markers, are maintained in

8 culture and differentiate under similar conditions, and share high transcriptome similarity. The

9 second is a CDX2+ putative human trophectoderm stem cell (hTESC) with distinct cell culture

10 requirements and differences in gene expression and differentiation relative to hTSCs. Derivation

11 of hTSCs and hTESCs from pluripotent stem cells significantly enables construction of models for

12 normal and pathological placental development.

\section{Keywords}

trophoblast stem cells, pluripotent stem cells, placenta, extravillous trophoblast, syncytiotrophoblast, embryonic stem cells, trophectoderm, trophectoderm stem cell, trophectodermal stem cell 


\section{Introduction}

Specification of the trophectoderm and the inner cell mass (ICM) is the first differentiation event during human embryonic development. The trophectoderm mediates blastocyst implantation in the uterus and is the precursor to all trophoblast cells in the placenta. Upon embryo implantation, the trophectoderm forms the cytotrophoblast (CTB), a putative stem cell that can differentiate to form the two major cell types in the placenta - the extravillous trophoblast (EVT) and the syncytiotrophoblast (STB) (Benirschke et al., 2012; Bischof and Irminger-Finger, 2005). The EVTs are involved in remodeling of uterine arteries, which is critical to ensure adequate perfusion of the placenta with maternal blood, whereas the multinucleated STB mediates the nutrient and gas exchange at the maternal-fetal interface (Moser et al., 2011; Yabe et al., 2016). Abnormalities in trophoblast development are associated with pregnancy-related pathologies such as miscarriage, preeclampsia and placenta accreta. Yet, despite its relevance to maternal and fetal health, constraints on research with human embryos and early fetal tissue impede mechanistic insight into early trophoblast development.

Trophoblast stem cells derived from first trimester human placental samples and blastocyst-stage embryos have emerged as an attractive in vitro model system for early human trophoblast (Okae et al., 2018). However, restricted accessibility of embryos and placental samples from early gestation and low genetic diversity of existing cell lines limit the use of this model. In contrast, pluripotent stem cells are a more accessible source for generation of in vitro models of human trophoblast. More importantly, unlike early gestation primary samples where the projected pregnancy outcome is uncertain, human induced pluripotent stem cells (hiPSCs) can potentially provide models of validated normal and pathological trophoblast development (Sheridan et al., 2019). However, whether bona fide trophoblast can be obtained from pluripotent stem cells has been a subject of intense debate (Roberts et al., 2014). A rigorous head-to-head comparison between trophoblast derived from pluripotent stem cells and their in vivo counterparts has proven 
difficult due to multiple reasons. Previous studies have used varying experimental protocols

(Roberts et al., 2018), a self-renewing trophoblast stem cell population has not been derived from

human pluripotent stem cells, and both primary placental samples and cultures of terminally dif-

ferentiated trophoblast obtained from pluripotent stem cells exhibit heterogeneity and contain many cell types.

In this study, we report the derivation and maintenance of two distinct trophectoderm lineage stem cells from human embryonic stem cells (hESCs) and hiPSCs in chemically defined culture conditions. The first is a CDX2- human trophoblast stem cell (hTSC) that is comparable to primary hTSCs derived from early gestation placental samples. The second is a more primitive CDX2+ cell type that is a putative human trophectoderm stem cell (hTESC) (Knöfler et al., 2019). Critically, the isolation of self-renewing stem cell populations allowed a direct comparison of primary hTSCs with pluripotent stem cell derived hTSCs; genome wide transcriptomic analysis and functional differentiation assays establish their equivalence. The routine derivation of hTSCs and hTESCs from pluripotent stem cells will provide powerful tools for mechanistic studies on normal and pathological early trophoblast development.

\section{Results}

\section{A chemically defined medium containing S1P enables differentiation of hESCs to CTB.}

Media formulations in previous studies on trophoblast differentiation of hESCs included signaling; YAP plays a critical role in specification of the trophectoderm in mouse (Knott and Paul,

61 2014; Nishioka et al., 2008; Yagi et al., 2007). We investigated the use of S1P in the context of 
trophoblast differentiation of hESCs under chemically defined culture conditions, by modifying our previous protocol that utilized KSR (Sarkar et al., 2015, 2016). H1 and H9 hESCs cultured in E8 medium were differentiated for 6 days in E7 medium (E8 without TGF $\beta 1$ ) supplemented with S1P, by treatment with BMP4 and the activin/nodal inhibitor SB431542 (Figure 1A). Under these conditions, we observed upregulation of the CTB markers CDX2 and ELF5 (Figure S1A, B). Upregulation of TBX4 was observed after 6 days. However, overall there were no significant changes in markers associated with neural or mesodermal differentiation after 6 days suggesting that differentiation to these lineages did not occur (Figure S1A, B). Immunofluorescence analysis at day 6 confirmed expression of the pan-trophoblast marker KRT7, and CTB markers P63 and GATA3 (Figure 1B; Figure S1C).

The putative CTB cells obtained at day 6 were investigated for their ability to differentiate to EVTs and STB, using protocols similar to those previously employed (Sarkar et al., 2015). Cells underwent an epithelial to mesenchymal transition over a 6-day period when passaged into E8 medium supplemented with epidermal growth factor (EGF) and SB431542. Immunofluorescence analysis showed expression of KRT7 and the EVT markers VE-Cadherin and HLA-G (Figure 1C, S1D). Alternatively, passaging CTB-like cells in E6 medium (E8 without TGF $\beta 1$ and bFGF) supplemented with activin and EGF resulted in formation of KRT7+ multinucleate cells expressing the STB markers hCG and syncytin over an 8-day period (Figure 1D, S1E). Removal of S1P from the medium during hESC differentiation to CTB-like cells abolished formation of EVTs that express HLA-G and VE-Cadherin (Figure 1E, S2A) under identical differentiation conditions (Figure 1A). Differentiation to STB also did not occur in the absence of S1P, as evidenced by lack of expression of syncytin and KRT7 (Figure 1F, S2B). Also, downregulation of the CTB marker CDX2 and upregulation of transcripts of neural and mesoderm markers was observed in cells after 6 days of differentiation, upon removal of S1P (Figure S2C). Taken together these results 
show that CTB-like cells - similar to those in previous studies utilizing more complex culture conditions (Sarkar et al., 2015) - can be obtained by differentiation of hESCs in a chemically defined medium containing S1P. Further, inclusion of S1P is necessary for hESC differentiation to trophoblast in our chemically defined culture medium.

Rho GTPase signaling, downstream of GPCRs activated by S1P, has been implicated in nuclear localization of YAP (Mo et al., 2012; Ohgushi et al., 2015). Both Rho/RhoA associated kinase (ROCK) and nuclear YAP play a critical role in trophectoderm specification in the mouse (Kono et al., 2014; Nishioka et al., 2009). Therefore, we investigated the role of Rho/ROCK signaling and YAP in trophoblast differentiation of hESCs. The Rho/ROCK inhibitor Y-27632 was included during differentiation of hESCs to CTB-like cells and subsequent differentiation to EVT and STB to investigate the role of Rho/ROCK signaling. Under these conditions, HLA-G expression was observed in cells obtained from H9 hESCs; however, VE-Cadherin expression was weak and observed in only a few cells (Figure S2D). On the other hand, expression of EVT markers was not observed in cells derived from H1 hESCs. Additionally, presence of ROCK inhibition abolished STB formation, as shown by lack of expression of syncytin and KRT7 (Figure S2E).

To investigate the role of YAP signaling in trophoblast differentiation of hESCs, we used an hESC cell line (H9) that expresses an inducible shRNA against YAP (H9-YAP-ishRNA) or a scrambled shRNA control (Hsiao et al., 2016). YAP knockdown abolished differentiation to EVT and STB, as evidenced by lack of expression of the relevant markers. Notably, high cell death was observed (Figure S2D, E). Gene expression analysis revealed significant reduction in ELF5 upon YAP knockdown, relative to the scrambled shRNA control (Figure S2F). Significant downregulation of the mesodermal genes $T B X 4$ and $L M O 2$ was observed, whereas $T$ was upregulated, in H9-YAP-ishRNA, relative to the scrambled control. Taken together, these results show that Rho/ROCK signaling, and YAP are necessary for differentiation of hESCs to functional CTB that can give rise to both EVTs and STB, in our chemically defined culture medium. 
A

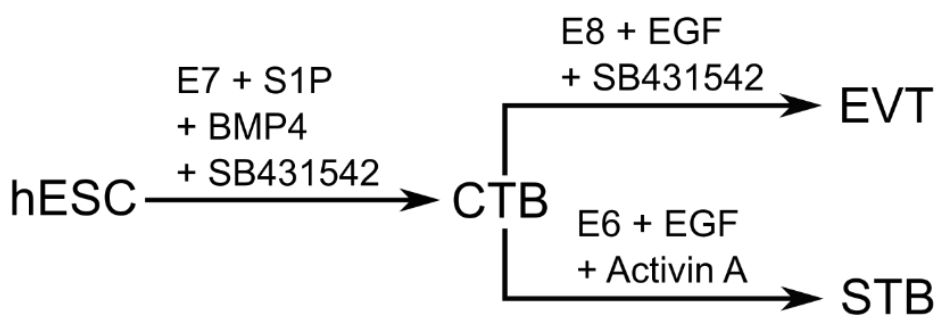

B 6 Day Treatment CTB

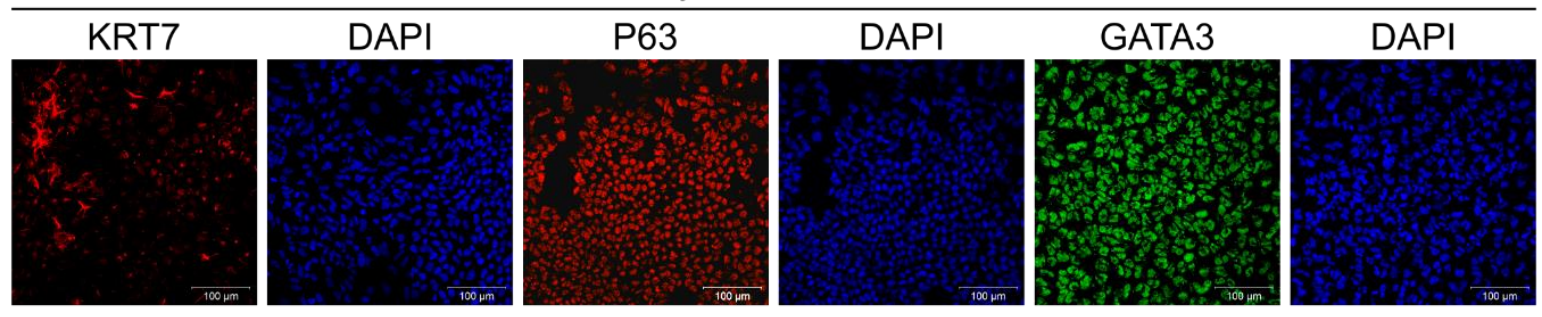

C 12 Day Treatment EVT

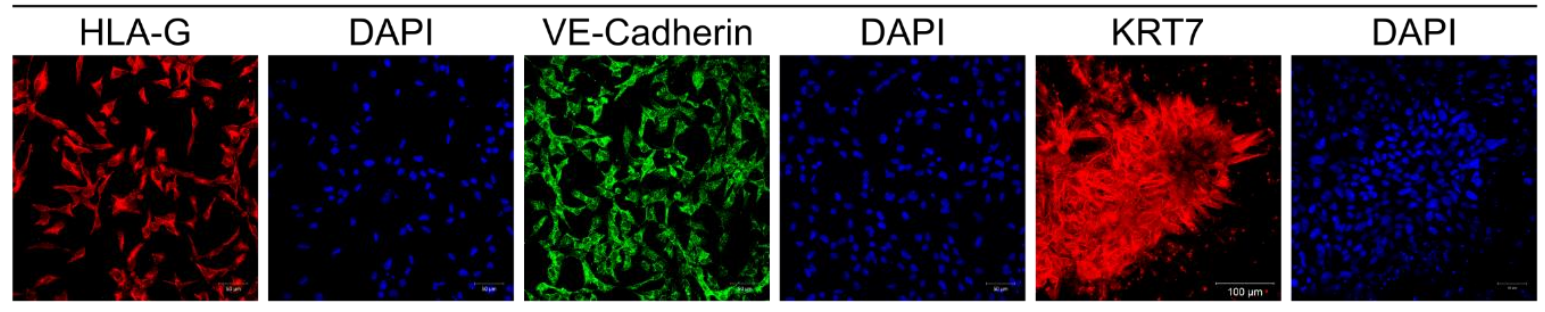

D

14 Day Treatment STB

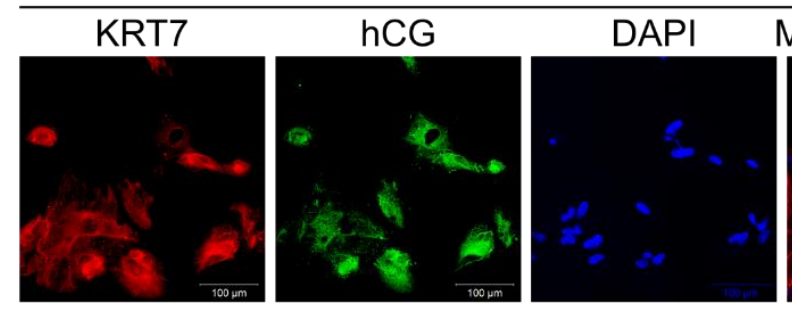

E

12 Day Treatment EVT (without S1P)
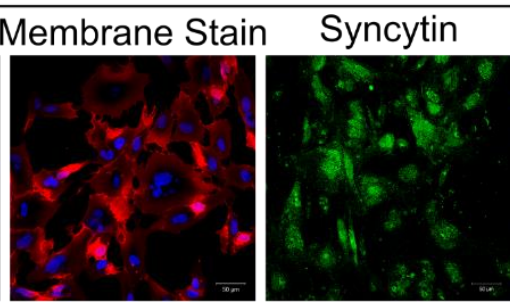

DAPI
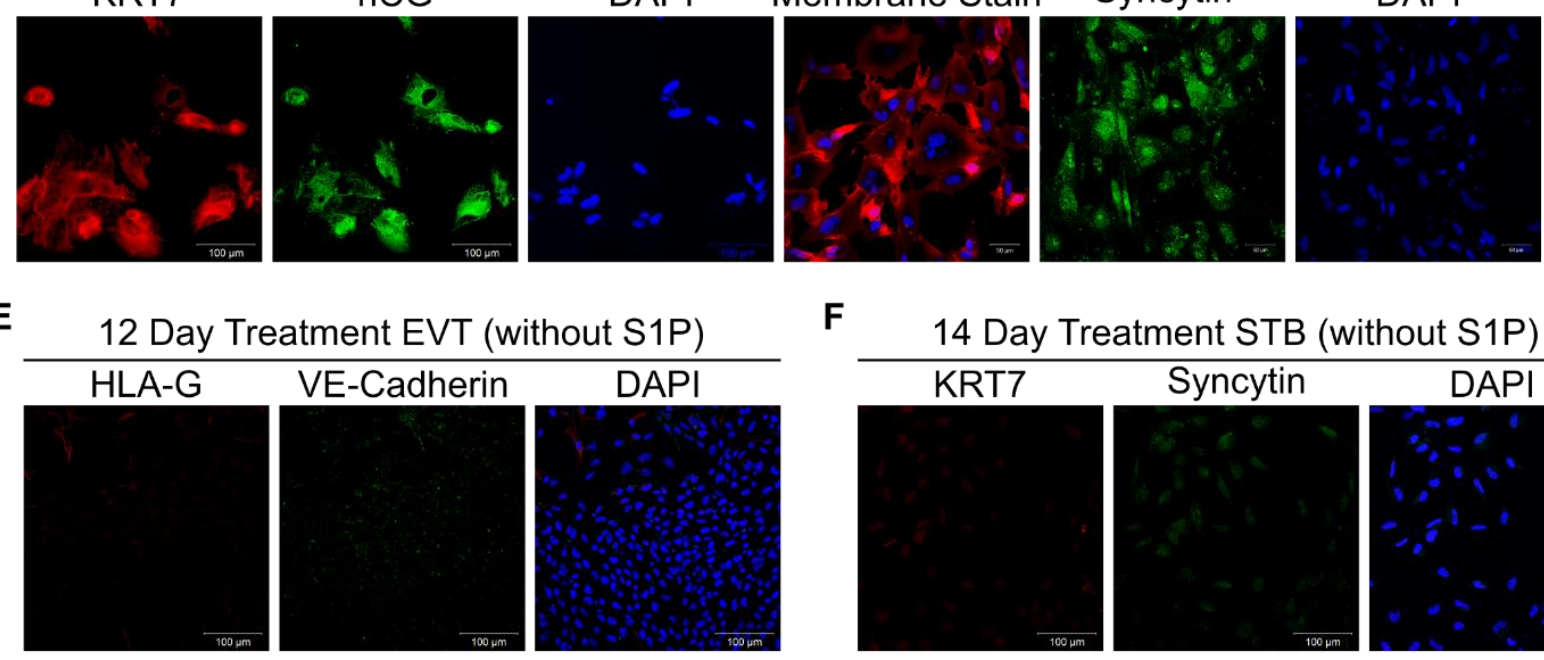

DAPI

F
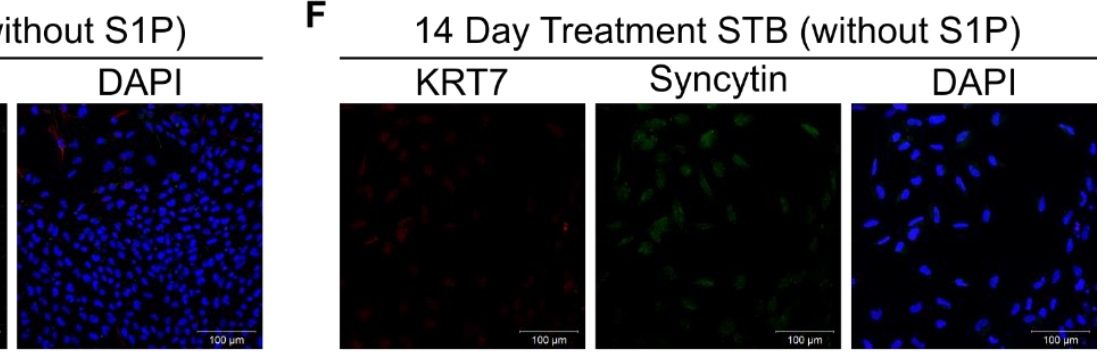


\section{Figure 1: A chemically defined medium containing S1P enables differentiation of hESCs} to CTB.

(A) Schematic of protocol for hESC differentiation to trophoblast.

(B) Immunostaining of KRT7, P63 and GATA3 in H9 hESCs at day 6 of initial treatment. Nuclei were stained with DAPI.

(C) Confocal images of EVTs from 12-day treatment of H9 hESCs, staining for KRT7, HLA-G and VE-Cadherin. Nuclei were stained with DAPI.

(D) Confocal images of STB from 14-day treatment of H9 hESCs, staining for KRT7, syncytin and hCG. Nuclei were stained with DAPI.

(E) Confocal images of cells from 12-day EVT treatment of H9 hESCs upon removal of S1P, staining for HLA-G and VE-Cadherin. Nuclei were stained with DAPI.

(F) Confocal images of cells from 14-day STB treatment of H9 hESCs upon removal of S1P staining for KRT7 and syncytin. Nuclei were stained with DAPI.

Scale bars are $100 \mu \mathrm{m}$ for all images.

S1P mediates its effects on trophoblast differentiation of hESCs through its receptors. hESC differentiation to trophoblast, we replaced S1P with D-erythro-dihydrospingosine-1-phosphate $(\mathrm{dhS1P})$ in our protocol. dhS1P acts as an agonist for the S1P receptors (S1PRs) but does not mediate an intracellular effect (Van Brocklyn et al., 1998). Replacing S1P with dhS1P yielded

117 similar results - CTB-like cells showed high expression levels of CDX2, GATA3, P63, and TEAD4

118 (Figure 2A; Figure S3A). Upon further differentiation as previously described (Figure 1A), STB expressing KRT7 and hCG, and EVT expressing HLA-G and VE-Cadherin were obtained (Figure

\section{B, C; Figure S3B, C).}


125 CYM5541, respectively - to replace S1P in differentiation protocols previously discussed. Expres126 sion of CDX2, GATA3, P63, and TEAD4 was observed in CTB-like cells for all three agonists

127 (Figure 2A; Figure S3A). Similarly, use of each agonist resulted in expression of the EVT mark128 ers HLA-G and VE-Cadherin, and formation of multinucleate STB expressing KRT7 and hCG 129 (Figure 2B, C; Figure S3B, C). However, we observed some variability between the agonists. 130 The intensity of CDX2 and P63 expression was higher with S1PR1 agonist CYM5442 and the 131 S1PR3 agonist CYM5541. Nuclear P63 expression was strongest for CYM5442 compared to 132 CYM5541. Notably, use of the S1PR2 agonist CYM5520 resulted in lower expression of CDX2, strong cytoplasmic expression of P63, and high heterogeneity in staining at day 6 relative to the other agonists. Formation of large multinucleated STB was more pronounced when the S1PR2 or S1PR3 agonists were used, as compared to the S1PR1 agonist. On the other hand, the S1PR1 and S1PR3 agonists enhanced formation of mesenchymal EVTs, relative to the S1PR2 agonist. sufficient for trophoblast differentiation of hESCs in our culture system. Since our qualitative observations showed that use of the S1PR3 agonist resulted in high CDX2 expression, and both 140 multinucleate STB and mesenchymal EVTs could be obtained when the S1PR3 agonist was 141 used, we chose the S1PR3 agonist for subsequent studies. 
bioRxiv preprint doi: https://doi.org/10.1101/762542; this version posted September 8, 2019. The copyright holder for this preprint (which was not certified by peer review) is the author/funder, who has granted bioRxiv a license to display the preprint in perpetuity. It is made available under aCC-BY-NC-ND 4.0 International license.

A

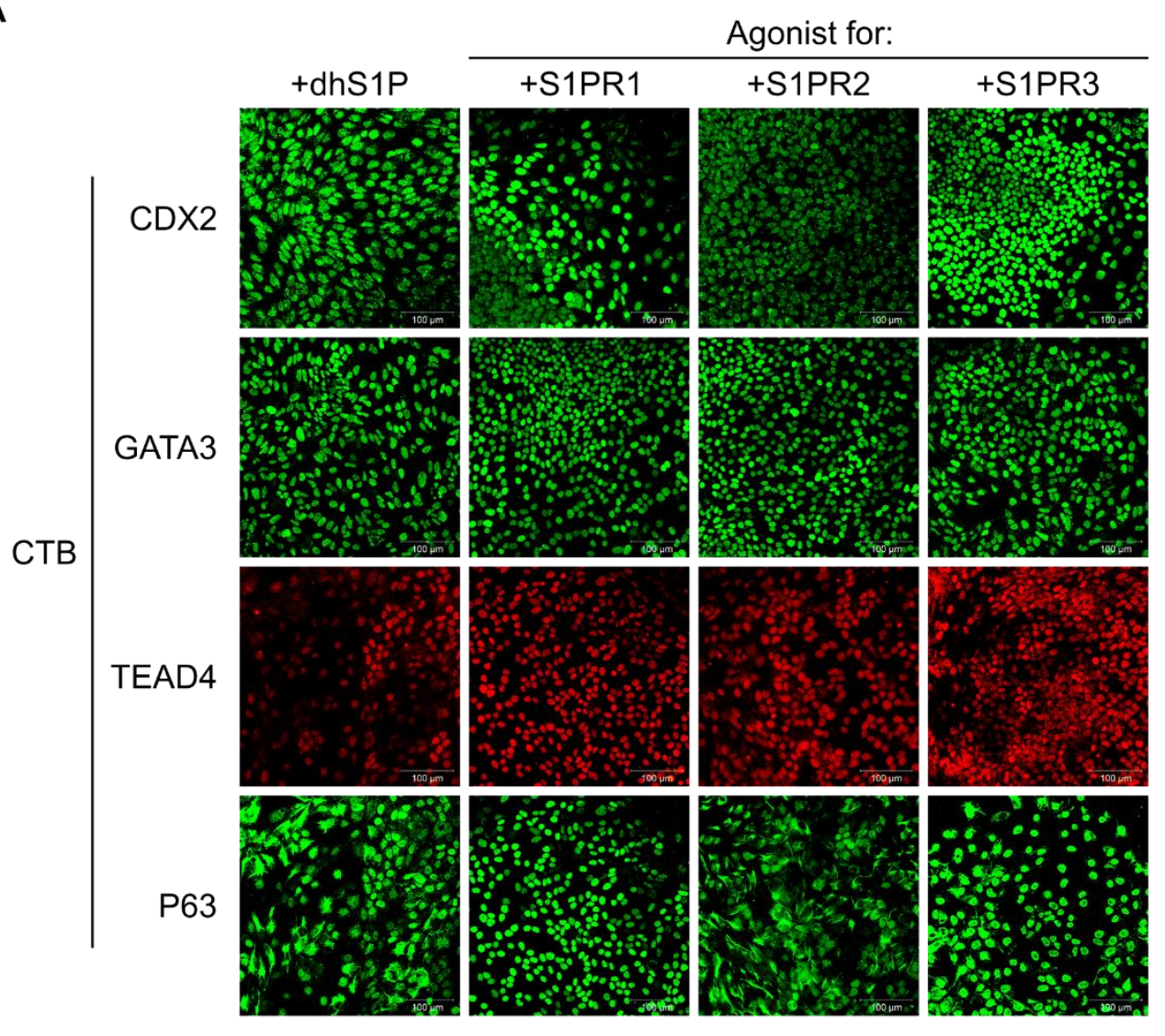

B

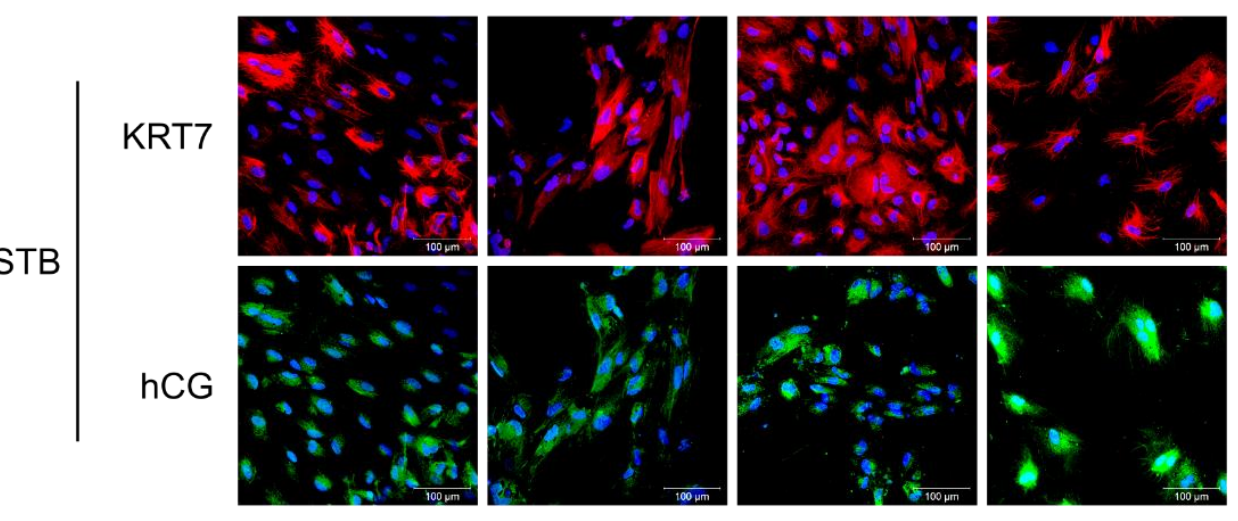

C

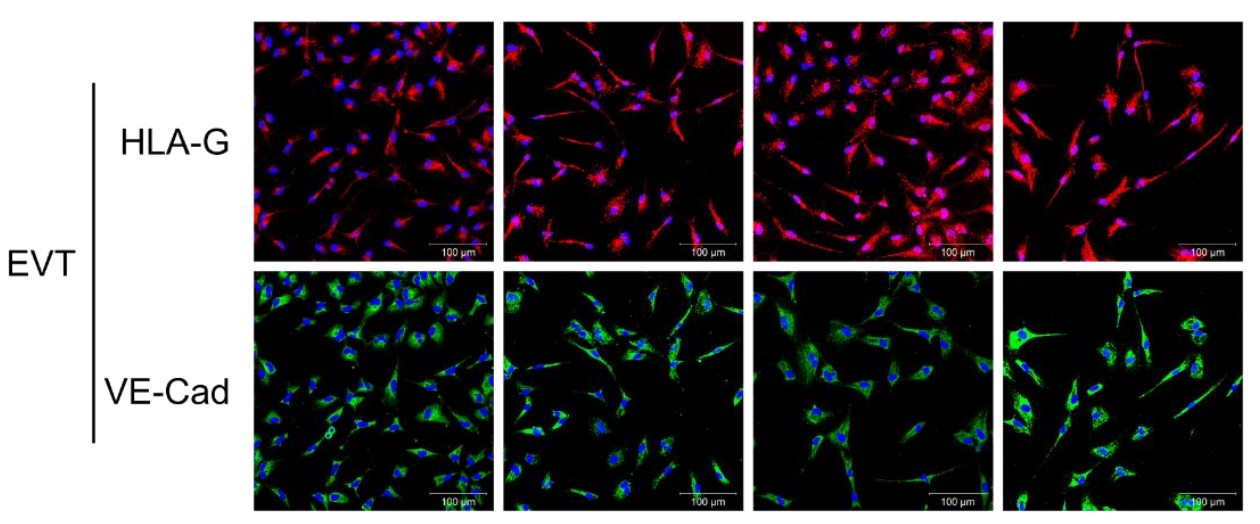


Figure 2: S1P mediates its effects on trophoblast differentiation of hESCs through its receptors.

(A) Confocal images of CTB-like cells from 6-day treatment of H9 hESCs using D-erythro-dihydrospingosine-1-phosphate (dhS1P), CYM5442 (S1PR1 agonist), CYM5220 (S1PR2 agonist), and CYM5541 (S1PR3 agonist), staining for CDX2, GATA3, P63, and TEAD4. Nuclei were stained with DAPI.

(B) Confocal images of STB cells from 14-day treatment of H9 hESCs using dhS1P, CYM5442, CYM5520, and CYM5541 during the initial 6-day treatment, staining for KRT7 and hCG. Nuclei were stained with DAPI.

(C) Confocal images of EVT cells from 12-day treatment of H9 hESCs using dhS1P, CYM5442, CYM5220, and CYM5541 during the initial 6-day treatment, staining for HLA-G and VE-Cadherin. Nuclei were stained with DAPI.

Scale bars are $100 \mu \mathrm{m}$ for all images.

\section{Optimizing timing of hESC differentiation enables derivation of CDX2+ $\mathrm{hTESCs.}$}

We investigated whether CTB-like cells obtained by treatment of hESCs with BMP4 and SB431542 in E7 medium supplemented with the S1PR3 agonist CYM5541 for 6 days could be passaged and maintained under conditions used for culture of primary hTSCs (Okae et al., 2018). Upon plating in trophoblast stem cell medium (TSCM) developed by Okae et al. (2018), hESCderived CTB-like cells underwent differentiation, and epithelial colonies could not be retained after a single passage. CDX2 expression is upregulated significantly in as little as 2 days after initiation of hESC differentiation (Figure S1A, B). Additionally, previous studies have reported differentia-

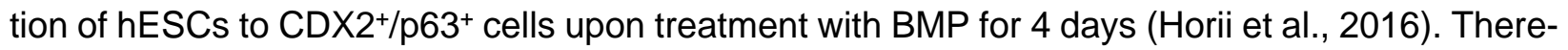
fore, we explored the use of a shorter differentiation step for obtaining CTB-like cells. After 3 days of differentiation, $\mathrm{H} 9$ and $\mathrm{H} 1$ hESCs expressed nuclear CDX2, P63, and TEAD4 uniformly (Figure 3B). Quantitative image analysis showed that nearly all cells are CDX2+ at day 3, in contrast to CTB-like cells at day 6. Notably, use of a 6-day protocol resulted in significantly reduced fraction CDX2+ cells in the case of H1 hESCs in comparison to H9 hESCs; H9 cells retained CDX2+ cells longer (Figure 3C). 
CDX2+ cells at day 3 were passaged into a chemically defined medium containing four major components (denoted TM4) - the S1PR3 agonist CYM5541, the GSK3 $\beta$ inhibitor CHIR99021, the TGF $\beta$ inhibitor A83-01, and FGF10. CHIR99021 and A83-01 are components of TSCM used for culture of primary hTSCs; FGF10 was included because FGFR2b signaling is active in primary hTSCs and the early placenta (Okae et al., 2018). Cells in TM4 could be maintained as epithelial colonies for $30+$ passages over the course of 5 months. In TM4 medium, cells derived from $\mathrm{H} 9$ and $\mathrm{H} 1$ hESCs expressed the trophoblast markers CDX2, TFAP2C, YAP, TEAD4, and GATA3 (Figure 3D; Figure S4A) (Choi et al., 2012; Home et al., 2012; Nishioka et al., 2008; Niwa et al., 2005; Ralston et al., 2010; Yagi et al., 2007). Additionally, cells expressed the pan-trophoblast marker KRT7, and low levels of P63, which is expressed in CTBs found in the placental villi. Notably, CDX2 expression has been strongly associated with the trophectoderm and is lost once placental villi are formed (Blakeley et al., 2015; Hemberger et al., 2010; Horii et al., 2016; Knöfler et al., 2019). Due to their expression of CDX2, and to distinguish them from trophoblast stem cells that do not express CDX2, these cells are denoted as human 171 trophectoderm stem cells (hTESCs).

\section{$\mathrm{P63}^{+} \mathrm{hTSC}$ derived from hESCs can be maintained in TSCM.}

We evaluated whether hTESCs could be maintained in TSCM used for culturing primary

174 hTSCs (Figure 3A) (Okae et al., 2018). When hTESCs cultured in TM4 for 5+ passages were

175 directly passaged into TSCM, cells underwent a change in colony morphology over 3 passages;

176 however, very little differentiation was observed. Notably, cell morphology of the hESC-derived 177 cells closely resembled that of primary hTSCs in TSCM (Figure S5A) (Okae et al., 2018). Strik178 ingly, however, hESC-derived hTESCs lost expression of CDX2 and gained high expression of 179 P63. As discussed earlier, cells could be maintained as epithelial colonies when hESCs after 3 days of differentiation were passaged into TM4. In contrast, passaging day-3 differentiated hESCs 
181 into TSCM resulted in extensive differentiation, although a few epithelial colonies could be ob-

182 served. Further passaging resulted in similar morphological changes in the epithelial colonies as

183 those observed for hTESCs transitioning to TSCM. After 6 passages, only epithelial colonies

184 remained, and they closely resembled both the hTESCs transitioned into TSCM and primary

185 hTSCs. H9 and H1 hESC-derived cells - passaged directly into TSCM after 3 days of differenti-

186 ation or transitioned from TM4 (Figure 3A) - showed high expression of YAP, TEAD4, TFAP2C,

187 and GATA3, similar to cells in TM4, but no expression of CDX2 (Figure 3E; Figure S4B). Lastly,

188 hESC-derived hTSCs exhibit similar expression profile of trophoblast markers as primary hTSCs

189 (Figure S5B). Therefore, these cells are denoted as hTSCs.

We further evaluated the differentiation potential of hESC-derived hTSCs using same protocols as those used by Okae et al. (2018) for differentiation of primary hTSCs to EVTs and STB

192 (Okae et al., 2018). Similar to primary hTSCs, the hESC-derived hTSCs could be differentiated 193 into mesenchymal EVTs expressing HLA-G and VE-Cadherin (Figure 3F; Figure S4C) and mul194 tinucleate cells expressing the STB markers hCG and KRT7 (Figure 3G; Figure S4D). Further, 195 hESC-derived hTSCs retained their ability to differentiate into STB- and EVTs after 30 passages 196 in TSCM. Strikingly however, hTESCs did not differentiate to EVTs using the same protocols used 197 for hTSCs (Figure S5C). Taken together along with differences in culture conditions for mainte198 nance and expression of the trophectoderm marker CDX2, these results suggest that hTESCs 199 and hTSCs represent two distinct stem cell populations, with hTESCs being a more primitive cell 200 type. 
A

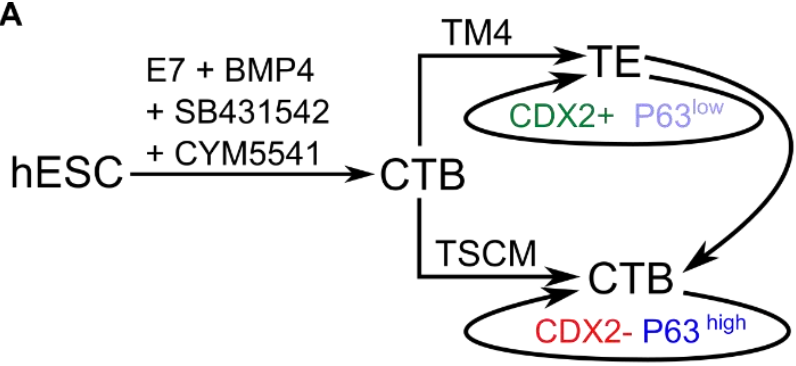

B

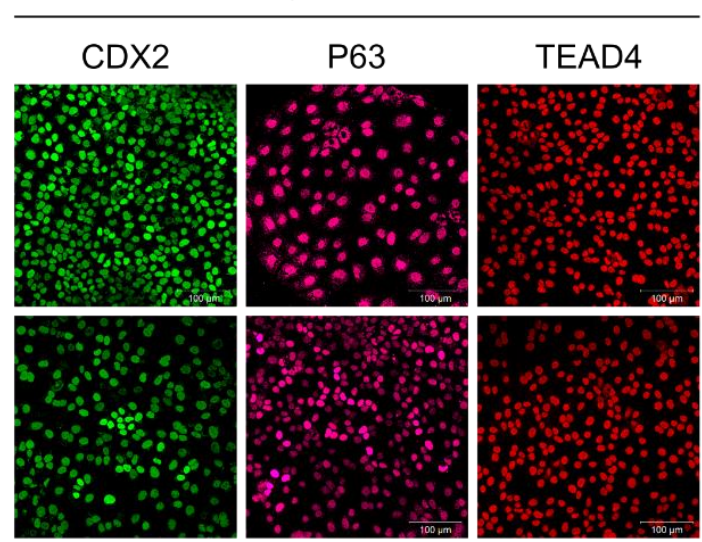

C

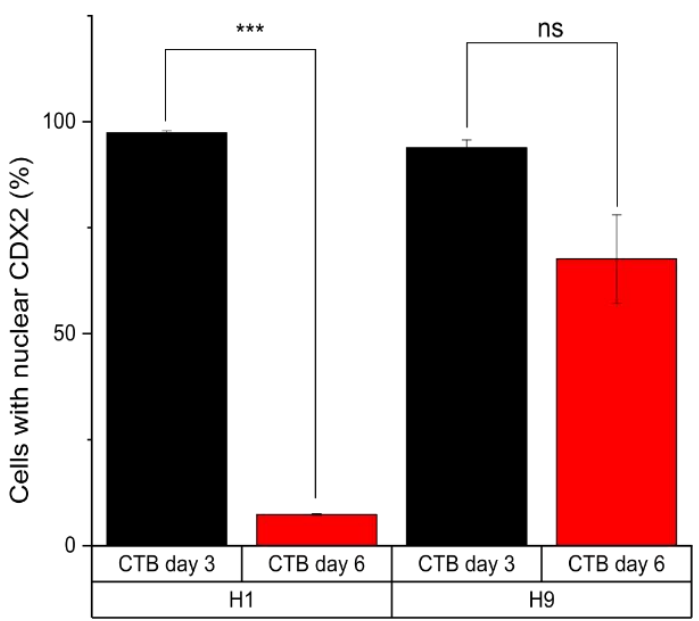

D

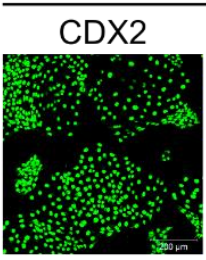

GATA3

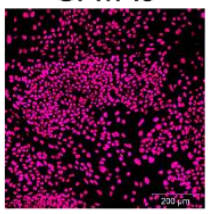

KRT7

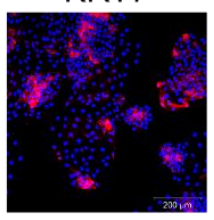

E hTSC in TSCM

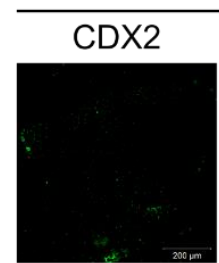

GATA3

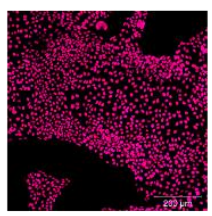

KRT7

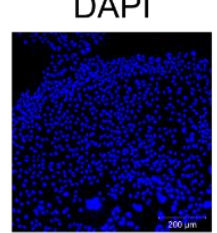

DAPI

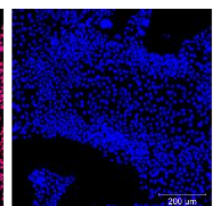

P63

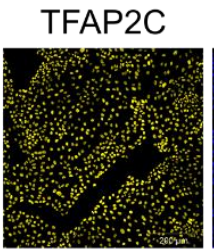

YAP

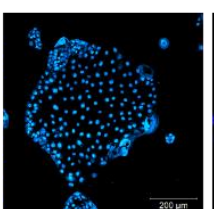

DAPI
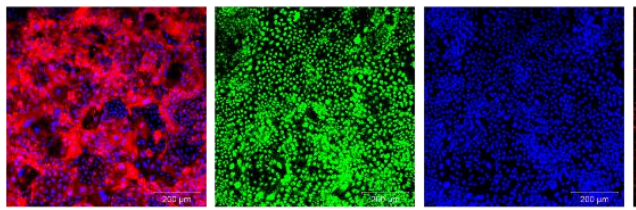

DAPI

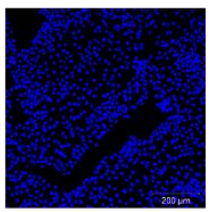

DAPI

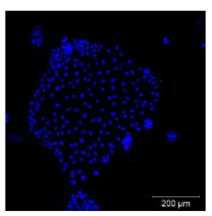

TEAD4

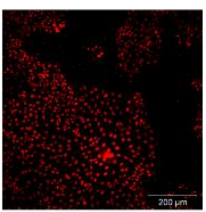

hTESC in TM4
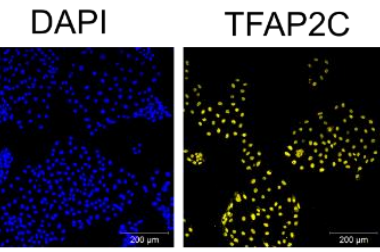

YAP
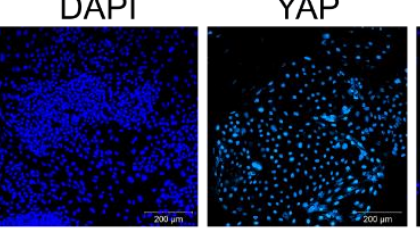

DAPI
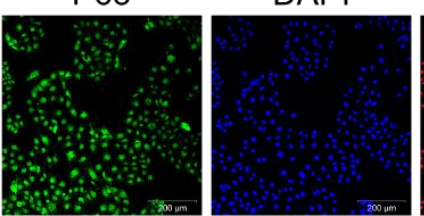

TEAD4

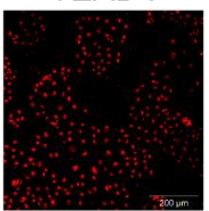

F

EVTs from hTSCs

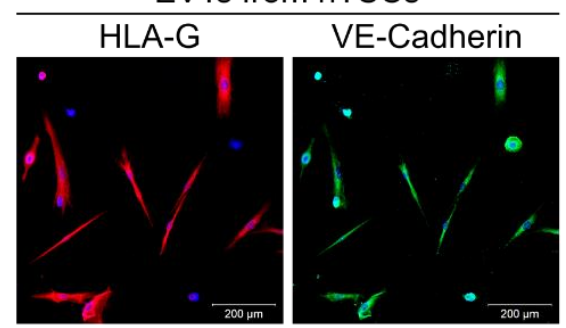

G

STB from hTSCs

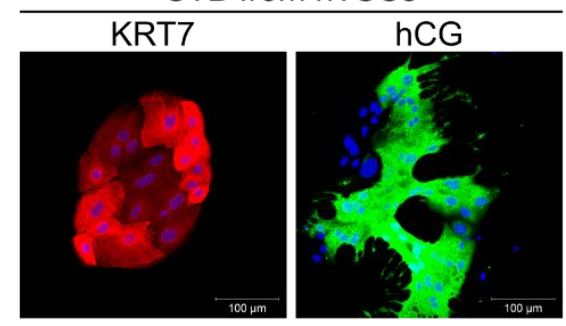


Figure 3: Optimizing timing of hESC differentiation enables derivation of CDX2+ hTESCs and P63+ hTSCs.

(A) Schematic of differentiation protocol for establishment of hTESCs and hTSCs from hESCs.

(B) Confocal images of 3-day treated $\mathrm{H} 9$ and $\mathrm{H} 1 \mathrm{hESCs}$, staining for CDX2, P63, and TEAD4. Nuclei were stained with DAPI. Scale bars are $100 \mu \mathrm{m}$.

(C) Quantitative analysis of cells expressing nuclear CDX2 after 3-day and 6-day differentiation of $\mathrm{H} 1$ (day $3, \mathrm{n}=5455$; day $6, \mathrm{n}=2448$ ) and $\mathrm{H} 9$ (day $3, \mathrm{n}=5552$; day $6, \mathrm{n}=6448$ ) hESCs. Analysis was performed in MATLAB and at least 2 biological replicates were used. (Error bars are S.E., *** $\mathrm{p}<0.05)$.

(D) Confocal images of H9 hESC-derived hTESCs in TM4, staining for CDX2, TFAP2C and GATA3, YAP, TEAD4, and P63. Nuclei were stained with DAPI. Scale bars are $200 \mu \mathrm{m}$.

(E) Confocal images of H9 hESC-derived hTSCs in TSCM, staining for CDX2, TFAP2C and GATA3, YAP, TEAD4, and P63. Nuclei were stained with DAPI. Scale bars are $200 \mu \mathrm{m}$.

(F) Confocal images of EVTs from H9 hESC-derived hTSCs, staining for HLA-G and VE-Cadherin. Nuclei were stained with DAPI. Scale bars are $200 \mu \mathrm{m}$.

(G) Confocal images of STB from H9 hESC-derived hTSCs staining for hCG and KRT7. Nuclei were stained with DAPI. Scale bars are $100 \mu \mathrm{m}$.

Transcriptome analysis confirms equivalence of hESC-derived and primary hTSCs and reveals differences between hTSCs and hTESCs

We conducted genome wide transcriptome analysis on hTESCs, and hESC-derived and primary hTSCs using RNA sequencing. Principal component analysis (PCA) of transcriptomic signatures showed that hESC-derived and primary hTSCs cluster together, indicating similarities in overall gene expression (Figure 4A). Hierarchical clustering analysis further confirmed the very 207 high transcriptome similarity between hESC-derived and primary hTSCs (Figure 4B). In conjunc208 tion with similarities in marker expression and culture conditions for maintenance and differentia209 tion, these results establish the equivalence of hESC-derived and primary hTSCs.

PCA also showed that hTESCs are a distinct population of cells that cluster differently

211 from hTSCs and hESCs differentiated to the trophoblast lineage for 3 days (Figure 4A). Higher expression of the trophectoderm-associated markers CDX2 and HAND1 is observed in hTESCs 
213 relative to hTSCs. On the other hand, expression of TP63 - associated with villous CTB - is

214 higher in hTSCs relative to hTESCs (Figure 4C). Statistical analysis of gene expression profiles

215 identified genes that were significantly differentially expressed between hTESCs and hTSCs.

216 Specifically, 269 genes showed significantly higher expression levels, and 275 genes showed

217 significantly lower expression levels in hTESCs vs. hTSCs (Tables S1 and S2). Gene set enrich-

218 ment analysis of these genes identified 300 and 47 gene ontology (GO) categories (out of 9996

219 queried categories) associated with genes showing higher and lower expression in hTESCs vs

220 hTSCs, respectively (Tables S3 and S4). Interestingly, consistent with differences in colony mor-

221 phology between hTESCs and hTSCs, genes associated with extracellular matrix, biological ad-

222 hesion, and cell-cell adhesion were upregulated in hTESCs. Taken together along with distinct

223 medium requirements for maintenance in cell culture, and differences in EVT differentiation under

224 identical assay conditions, these results show that hTSCs and hTESCs represent distinct stem

225 cell populations. 
bioRxiv preprint doi: https://doi.org/10.1101/762542; this version posted September 8, 2019. The copyright holder for this preprint (which was not certified by peer review) is the author/funder, who has granted bioRxiv a license to display the preprint in perpetuity. It is made available under aCC-BY-NC-ND 4.0 International license.

A

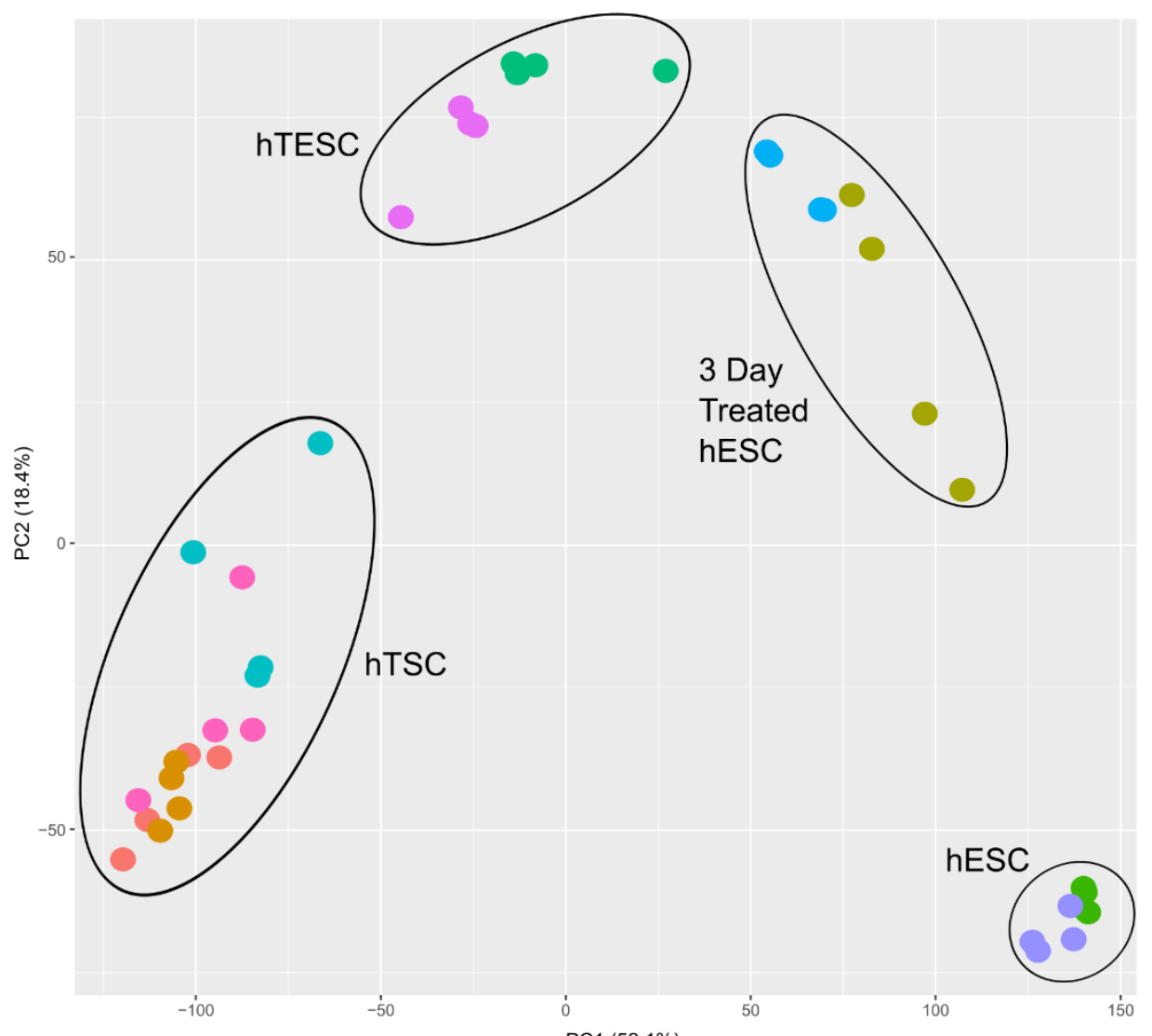

Group

CT29 hTSCs TSCM

CT30 hTSCs TSCM

H1 hESC 3 Day Treatment

H1 hESC E8

H1 hTESCS TM4

$\mathrm{H} 1 \mathrm{hTSC}$ TSCM

H9 hESC 3 Day Treatment

H9 hESC E8

H9 hTESCs TM4

H9 hTSCs TSCM

B

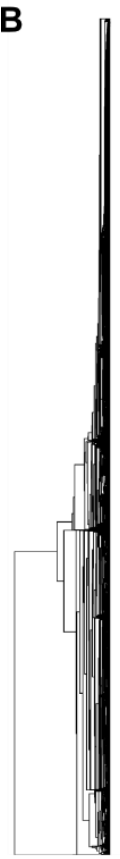

PC1 (52.1\%)

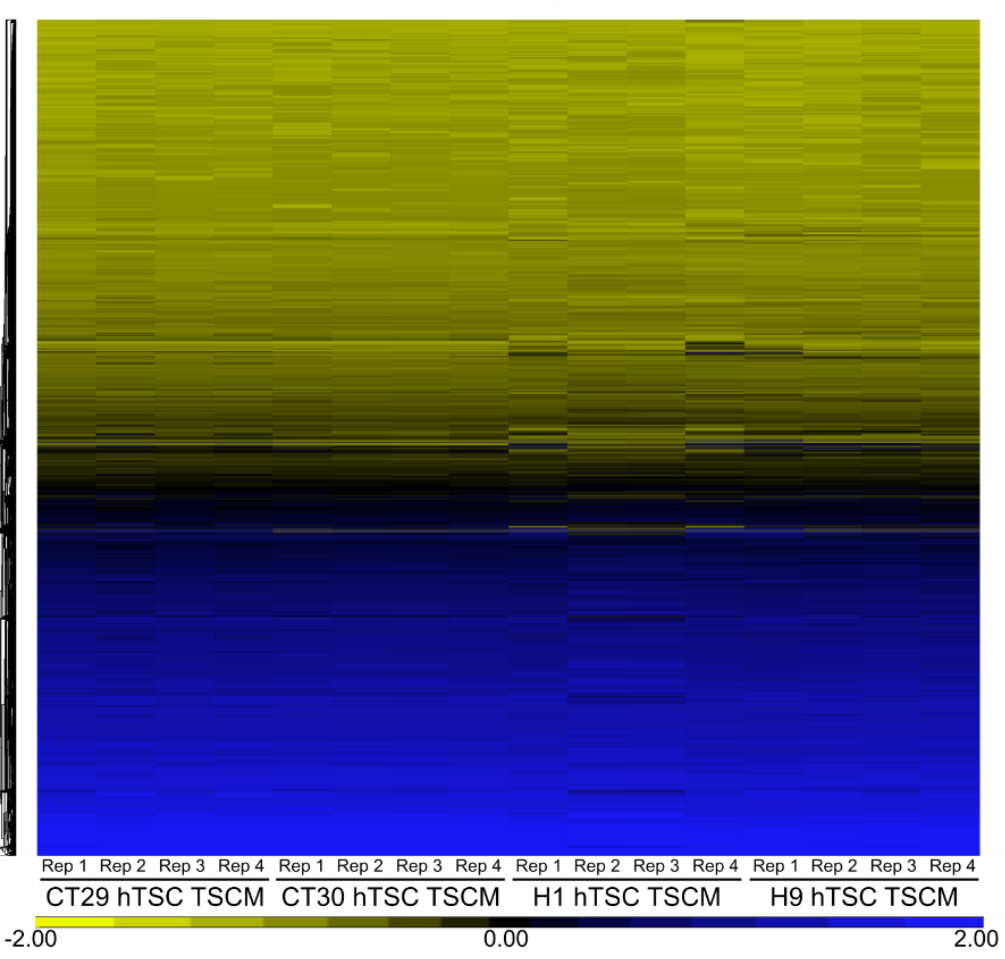

C
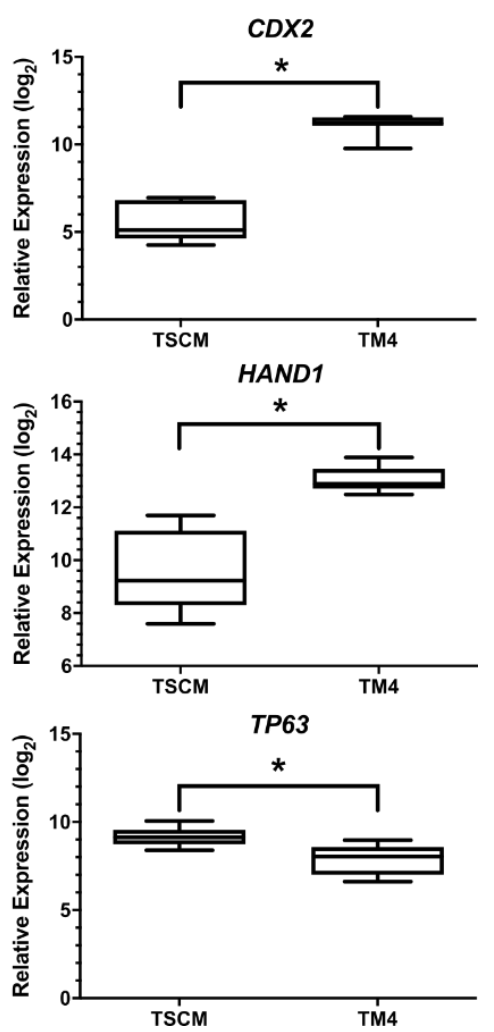
Figure 4: Transcriptome analysis confirms equivalence of hESC-derived and primary hTSCs and reveal differences between hTSCs and hTESCs

(A) Principal component analysis (PCA) of transcriptome data on $\mathrm{H} 1$ and $\mathrm{H} 9 \mathrm{hESCs}, \mathrm{H} 1$ and $\mathrm{H} 9$ hESCs after 3-day treatment, $\mathrm{H} 1$ and $\mathrm{H} 9$ hESC-derived hTESCs cultured in TM4, $\mathrm{H} 1$ and $\mathrm{H} 9$ hESC-derived and primary (CT29 and CT30) hTSCs cultured in TSCM.

(B) Hierarchical clustering analysis of transcriptome data from $\mathrm{H} 1$ and $\mathrm{H} 9 \mathrm{hESC}$-derived and primary (CT29 and CT30) hTSCs.

(C) Relative expression of trophectoderm-associated markers CDX2 and HAND1 and villous CTB-associated marker TP63 in $\mathrm{H} 1$ and H9 hESC-derived hTESCs and hTSCs ( $\left.{ }^{*} \mathrm{q}<0.001\right)$.

HTESCs and hTSCs can be generated from hiPSCs.

Lastly, we investigated if our results on derivation of hTESCs and hTSCs from hESCs can be extended to hiPSCs. Accordingly, we used our previously described protocols (Figure 3A) to derive hTESCs and hTSCs from the hiPSC line SBli006-A. HTESCs derived from SBli006-A hiPSCs maintained expression of CDX2, TFAP2C, GATA3, YAP KRT7, and TEAD4, along with low expression level of P63 in TM4 (Figure 5A). Similarly, hTSCs derived from SBli006-A hiPSCs expressed KRT7, P63, TEAD4, TFAP2C, YAP, and GATA3 in TSCM (Figure 5B). Similar to the case with hESC-derived hTSCs, cells lost expression of CDX2 but gained higher expression levels of P63 and KRT7 in TSCM. Differentiation of hTSCs derived from SBli006-A hiPSCs using protocols described by Okae et al. (2018), resulted in formation of mesenchymal EVTs with high expression of HLA-G and VE-Cadherin (Figure 5C), and multinucleate STB expressing hCG and KRT7 (Figure 5D). These results show that hTESCs and hTSCs can also be derived from hiPSCs. 
A hTESC in TM4

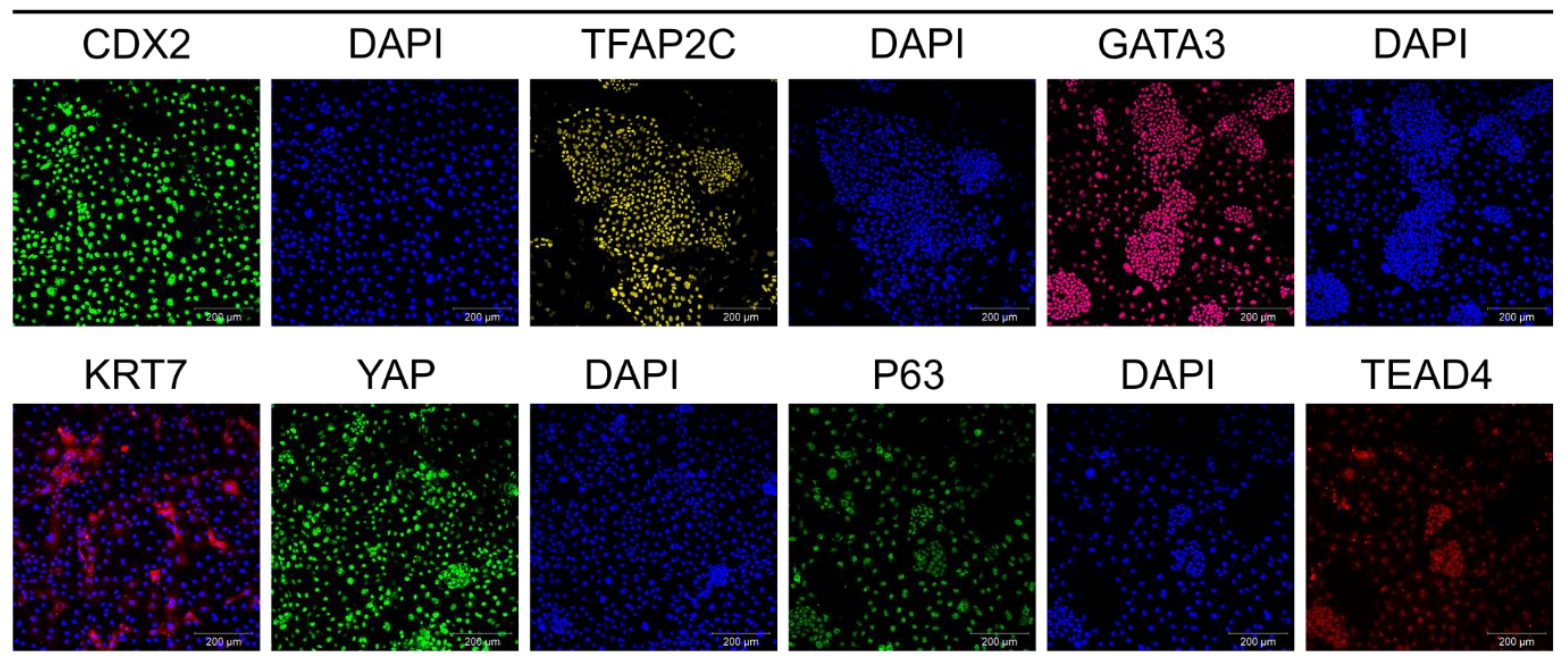

B hTSCs in TSCM

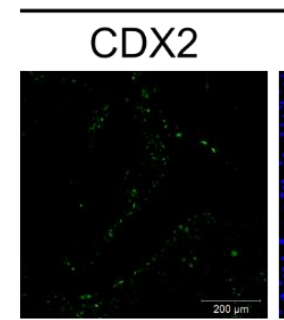

KRT7
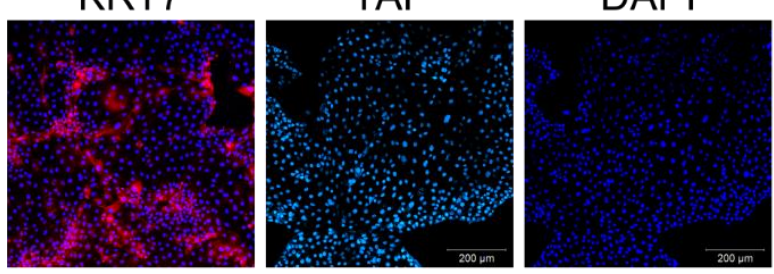

C

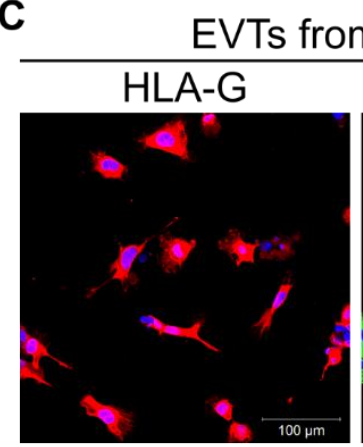

DAPI

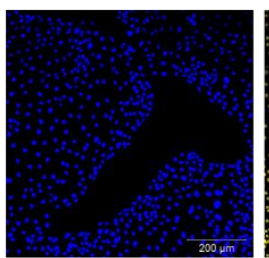

YAP

hTSCs

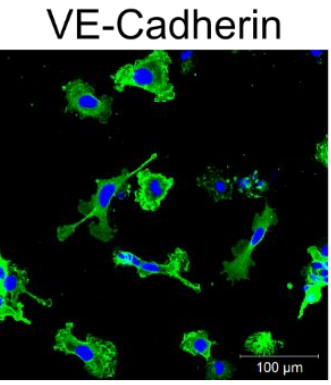

D

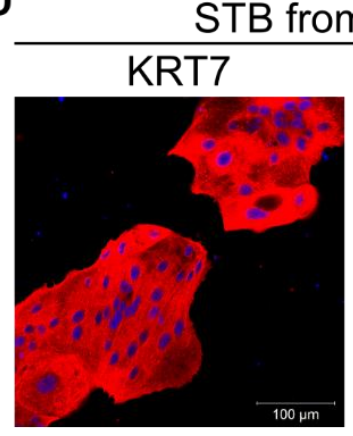

DAPI

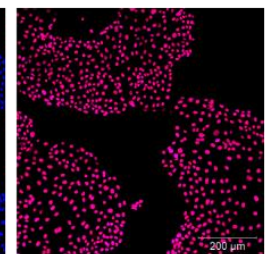

DAPI
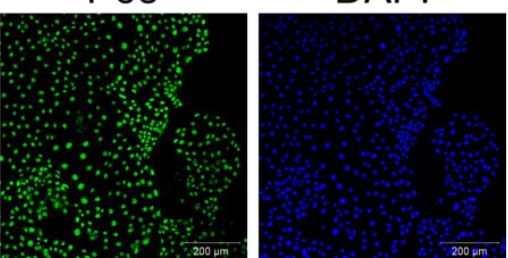

TEAD4
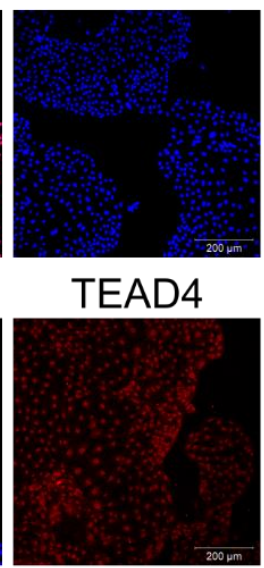

hs

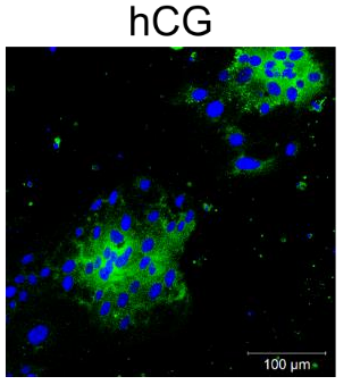


Figure 5: hTESCs and hTSCs generated from human iPSCs.

(A) Confocal image of SBli006-A-derived hTESCs in TM4, staining for CDX2, TFAP2C, GATA3, YAP, TEAD4, and P63. Nuclei were stained with DAPI. Scale bars are $200 \mu \mathrm{m}$.

(B) Confocal images of SBli006-A-derived hTSCs in TSCM, staining for CDX2, TFAP2C, GATA3, YAP, TEAD4, and P63. Scale bars are $200 \mu \mathrm{m}$.

(C) Confocal images of EVTs from SBli006-A-derived hTSCs, staining for HLA-G and VE-Cadherin. Scale bars are $100 \mu \mathrm{m}$.

(D) Confocal images of STB from SBli006-A-derived hTSCs, staining for hCG and KRT7. Scale bars are $100 \mu \mathrm{m}$.

In this study, we have shown that two distinct stem cell populations of the trophectoderm lineage - hTSCs and hTESCs - can be derived from hESCs and hiPSCs under chemically defined culture

242 conditions. Whether bona fide trophoblast can be obtained from human pluripotent stem cells

243 has been a subject of debate (Roberts et al., 2014). Despite extensive research in this area,

244 conducting a rigorous head-to-head comparison between hESC-derived and primary trophoblasts

245 has been challenging. The isolation of trophoblast stem cell populations from hESCs in this study,

246 in conjunction with the recent derivation of primary hTSCs (Okae et al., 2018) enabled such a

247 comparison. We have shown that hESCs can be differentiated to hTSCs that express markers 248 consistent with primary hTSCs (P63, TEAD4, TFAP2C, YAP, and GATA3). The hESC-derived

249 hTSCs are cultured in the same medium as primary hTSCs and differentiate to EVT and STB 250 using similar protocols as those used for primary hTSCs. Further, hESC-derived hTSCs and pri-

251 mary hTSCs have highly similar transcriptomes. Taken together, these results establish the equiv-

252 alence of hESC-derived and primary hTSCs and demonstrate that hESCs can indeed differentiate 253 to bona fide trophoblasts. 

entiation of hESCs.

Previous studies on trophoblast differentiation of hESCs have employed differing protocols, resulting in significantly different outcomes in some cases. Notably, Bernardo et al. (2011) reported that BMP treatment of hESCs results in differentiation of hESCs to mesoderm and not trophoblast. Our studies suggest two potential explanations for discrepancies in previous studies. First, our results show that receptor-mediated signaling by the albumin-associated sphingolipid S1P plays a critical role in hESC differentiation to trophoblast in our medium. Differences in results reported by previous studies may be due to variability in the lipid composition of media used during trophoblast differentiation of hESCs. Second, the medium used for routine maintenance of undifferentiated hESCs likely contributes significantly to their differentiation potential. For instance, unlike hESCs cultured in the presence of KSR, hESCs in E8 medium exhibit features of naïve pluripotency (Cornacchia et al., 2019). Recent studies report the conversion of hESCs to expanded potential stem cells (EPSCs) by transitioning hESCs to a human EPSC medium. Significantly, hTSClike cells can be obtained by passaging EPSCs - but not hESCs in KSR containing medium - in TSCM used for culture of primary hTSCs (Gao et al., 2019). The efficiency of establishing hTSClike lines was low ( 30\% with manual isolation of colonies) and a rigorous transcriptome com-

271 parison with primary hTSCs was not conducted. Nevertheless, taken together these studies un-

272 derscore the importance of hESC culture conditions in their differentiation potential. Differences

273 in culture conditions for undifferentiated hESCs may lead to inconsistent results during trophoblast 274 differentiation of hESCs. entiation of hESCs in our medium. However, the exact molecular mechanisms that underlie ac-

277 quisition of trophoblast fate in the presence of S1P receptor activation need to be further studied. 
278

279

280

281

282

283

284

285

286

287

288

289

290

291

292

293

294

295

296

297

Our results do not preclude the possibility that Rho/ROCK and/or YAP acts independently of S1P receptor mediated signaling in our system.

\section{Differences between hTESCs and hTSCs}

Marker expression analysis, functional differentiation assays, and genome-wide transcriptome analysis confirm the equivalence of hESC-derived hTSCs and primary hTSCs that are similar to villous CTB. However, hTESCs differ significantly from hTSCs. They do not undergo differentiation to EVTs under the culture conditions used for differentiating hESC-derived and primary hTSCs. Transcriptome analysis shows that genes associated with several key pathways and biological processes are differentially regulated between hTESCs and hTSCs. Significantly, hTESCs - but not hTSCs - express high levels of the trophectoderm-associated markers CDX2 and HAND1. Therefore we opt for the nomenclature human trophectoderm stem cells (hTESCs) as proposed by Knöfler et al. (2019), to distinguish these CDX2+ stem cells from hTSCs. Consistent with the more primitive nature of hTESCs, they can be readily transitioned into TSCM used for culturing hTSCs, as was seen by Okae et al. (2018) when transitioning trophectoderm cells of blastocysts into TSCM. Subsequently, similar to primary hTSCs, hTESCs lose expression of CDX2 and express higher levels of P63 in TSCM, and can differentiate to form EVTs and STB. Note that primary hTSCs derived from the trophectoderm in the blastocyst stage embryo lose expression of CDX2 in TSCM (Okae et al., 2018). On the other hand, it has not been possible yet to revert hTSCs to hTESCs by culturing in TM4 medium. Further studies are needed to rule out the possibility of such a reversion to the more primitive cell type.

\section{Considerations for derivation and culture of hTESCs}

To derive hTESCs, undifferentiated hESCs maintained in E8 medium are first treated for 3 days with the S1PR3 agonist, BMP4 and the activin/nodal inhibitor SB4315432, to obtain CDX2+ cells. Subsequently, CDX2+ cells are passaged in TM4 medium to obtain hTESCs. Using this protocol, 
we observed increased differentiation of $\mathrm{H} 1$-derived cells upon passage into TM4 medium, relative to H9- and SBli006-A-derived cells. Shortening the initial treatment step in case of H1 hESCs to 2 days eliminated excessive differentiation and facilitated derivation of hTESCs. However, we were unable to derive hTESCs with any cell line when the initial treatment was greater than 3 days.

In our studies, the initial hESC differentiation step was carried out in E7 medium that contains bFGF. Differentiation of hESCs to trophoblast has been carried out in the presence or absence of exogenous FGF (Amita et al., 2013; Das et al., 2007). Consistent with this, we found that hTESCs could be formed even if the initial treatment was carried out in E6 medium lacking bFGF, instead of E7 medium.

It is important to note that hTESCs proliferate slower in culture than hTSCs. We also observe that the attachment of hTESCs to tissue culture plates is less efficient than hTSCs. Finally, we observe that excessive differentiation in TM4 medium during early passages could be countered by reducing the concentration of ascorbic acid (32 $\mu \mathrm{g} / \mathrm{mL}$ instead of $64 \mu \mathrm{g} / \mathrm{mL}$ ) in TM4. Additional studies on composition of TM4 medium or the substrates used to coat tissue culture plates may lead to improved growth rate and attachment efficiency. Alternatively, the slower growth rate and less efficient attachment characteristics may be an inherent feature of the hTESC state. Nonetheless, we have successfully maintained hTESCs derived from all cell lines studied for at least 20 passages, in several independent runs over $5+$ months. We recommend passaging hTESCs routinely at higher cell densities relative to hTSCs, and troubleshooting cell line specific variability by optimizing the initial treatment step and/or lowering ascorbic acid concentration in TM4. 


\section{Derivation of hTSCs from hiPSCs}

We have shown that hTESCs and hTSCs can be derived from hiPSCs. Since hiPSCs can be derived by reprogramming easily accessible somatic tissues, hTSCs and hTESCs derived

327 from hiPSCs can greatly accelerate research in placental biology. Further, arguably a limitation of primary hTSCs is that pregnancy outcomes at term for the early gestation placental samples or blastocyst stage embryos used cannot be predicted accurately. In contrast, hiPSC-derived hTSCs and hTESCs, from hiPSCs generated using somatic tissues obtained at term, will enable development of models of validated normal and pathological trophoblast development. Pertinently, Sheridan et al. (2019) have derived hiPSCs from umbilical cords of normal pregnancies

337 from human pluripotent stem cells. These stem cell models will be powerful tools for in vitro 338 studies on human trophoblast development.

\section{Materials and Methods}

\section{Key resources table}

\begin{tabular}{|l|l|l|}
$\begin{array}{l}\text { REAGENT or RESOURCE } \\
\text { Antibodies }\end{array}$ & SOURCE & IDENTIFIER \\
\hline Anti-KRT7 & Santa Cruz Biotechnology & Cat\#sc-23876, RRID:AB_2265604 \\
\hline Anti-KRT7 & Cell Signaling Technologies & Cat\# 4465, RRID:AB_11178382 \\
\hline Anti-hCG & Abcam & Cat\# ab9582, RRID:AB_296507 \\
\hline Anti-hCG & Abcam & Cat\# ab9376, RRID:AB_307221 \\
\hline Anti-P63 & Cell Signaling Technologies & Cat\# 13109, RRID:AB_2637091 \\
\hline Anti-GATA3 & Cell Signaling Technologies & Cat\# 5852, RRID:AB_10835690 \\
\hline Anti-TFAP2C & Cell Signaling Technologies & Cat\# 2320, RRID:AB_2202287 \\
\hline Anti-YAP & Cell Signaling Technologies & Cat\# 4912, RRID:AB_2218911 \\
\hline Anti-TEAD4 & Abcam & Cat\# ab58310, RRID:AB_945789 \\
\hline
\end{tabular}




\begin{tabular}{|c|c|c|}
\hline Anti-CDX2 & Abcam & Cat\# ab76541, RRID:AB_1523334 \\
\hline Anti-VE-Cadherin & Cell Signaling Technologies & Cat\# 2500, RRID:AB_10839118 \\
\hline Anti-HLA-G & Abcam & Cat\# ab52455, RRID:AB_880552 \\
\hline Anti-Syncytin & Santa Cruz Biotechnology & Cat\# sc-50369, RRID:AB_2101536 \\
\hline Rabbit Polyclonal IgG & R\&D Systems & Cat\# AB-105-C, RRID:AB_354266 \\
\hline Rabbit XP IgG & Cell Signaling Technologies & Cat\# 3900, RRID:AB_1550038 \\
\hline Mouse IgG1 & Abcam & Cat\# ab18447, RRID:AB_2722536 \\
\hline Mouse IgG2a & Abcam & Cat\# 554126, RRID:AB_479661 \\
\hline $\begin{array}{l}\text { Alexa Fluor 488-conjugated } \\
\text { anti-rabbit IgG }\end{array}$ & Thermo Fisher Scientific & Cat\# A-11034, RRID:AB_2576217 \\
\hline $\begin{array}{l}\text { Alexa Fluor 647-conjugated } \\
\text { anti-rabbit IgG }\end{array}$ & Thermo Fisher Scientific & Cat\# A-21052, RRID:AB_2535719 \\
\hline DAPI & R\&D Systems & Cat\#5748 \\
\hline \multicolumn{3}{|c|}{ Chemicals, Peptides, and Recombinant Proteins } \\
\hline TrypLE & Thermo Fisher Scientific & Cat\#12604013 \\
\hline Vitronectin & Thermo Fisher Scientific & Cat\#A14700 \\
\hline Laminin 521 & Stem Cell Technologies & Cat\#77003 \\
\hline TeSR-E8 & Stem Cell Technologies & Cat\#05990 \\
\hline TeSR-E7 & Stem Cell Technologies & Cat\#05914 \\
\hline TeSR-E6 & Stem Cell Technologies & Cat\#05946 \\
\hline ReLeSR & Stem Cell Technologies & Cat\#05872 \\
\hline Sphingosine-1-phosphate & Tocris & Cat\#1370 \\
\hline $\begin{array}{l}\text { D-erythro-dihydrosphingosine-1-phos- } \\
\text { phate }\end{array}$ & Abcam & Cat\#ab141750 \\
\hline SB431542 & Tocris & Cat\#1614 \\
\hline BMP4 & Thermo Fisher Scientific & Cat\#PHC9534 \\
\hline CYM5442 hydrochloride & Tocris & Cat\#3601 \\
\hline CYM5520 & Tocris & Cat\#5418 \\
\hline CYM5541 & Tocris & Cat\#4897 \\
\hline Y-27632 dihydrochloride & Tocris & Cat\#1254 \\
\hline EGF & R\&D Systems & Cat\#236-EG \\
\hline Doxycycline hyclate & Tocris & Cat\#4090 \\
\hline Puromycin dihydrochloride & Tocris & Cat\#4089 \\
\hline Activin A & R\&D Systems & Cat\#338-AC \\
\hline Greiner Bio-one Cell View glass plates & Greiner Bio-one & Cat\#627965 \\
\hline 4\% Paraformaldehyde in PBS & Thermo Fisher Scientific & Cat\#R37814 \\
\hline Triton $\mathrm{X}-100$ & Sigma & Cat\#T8787 \\
\hline PBS w/o CaMg & Sigma & Cat\#D5773 \\
\hline PBS w/ CaMg & Sigma & Cat\#D8662 \\
\hline Human IgG & Immunoreagents & Cat\#Hu-003-C \\
\hline BSA & Fisher Scientific & Cat\#BP9703 \\
\hline $10 \%$ BSA fatty acid free in PBS & Sigma & Cat\#A1595 \\
\hline VPA & Sigma & Cat\#P6273 \\
\hline A83-01 & Tocris & Cat\#2939 \\
\hline
\end{tabular}




\begin{tabular}{|c|c|c|}
\hline 2-mercaptoethanol & Sigma & Cat\#M3148 \\
\hline FBS & Thermo Fisher Scientific & Cat\#16141-061 \\
\hline DMEM/F12 & Thermo Fisher Scientific & Cat\#11320033 \\
\hline ITS-X & Thermo Fisher Scientific & Cat\#51500-056 \\
\hline L-ascorbic acid & Sigma & Cat\#A8960 \\
\hline Pen/Strep & Thermo Fisher Scientific & Cat\#15140122 \\
\hline Forskolin & Tocris & Cat\#1099 \\
\hline Neuregulin & Cell Signaling Technologies & Cat\#5218SC \\
\hline Matrigel & Corning & Cat\#354234 \\
\hline KSR & Thermo Fisher Scientific & Cat\#10828028 \\
\hline Trizol Reagent & Thermo Fisher Scientific & Cat\#15596018 \\
\hline DEPC & Sigma & Cat\#95284 \\
\hline Baseline Zero DNAase Kit & VWR & Cat\#76081-624 \\
\hline Oligo-dT & IDT & Cat\#51-01-15-07 \\
\hline dNTP mix & Thermo Fisher Scientific & Cat\#10297018 \\
\hline Superscript II RT & Thermo Fisher Scientific & Cat\#18064014 \\
\hline SYBR Green Supermix & Bio-rad & Cat\#1725272 \\
\hline Methanol & Fisher Scientific & Cat\#A412-500 \\
\hline Acetone & Fisher Scientific & Cat\#A18-500 \\
\hline \multicolumn{3}{|l|}{ Critical Commercial Kits } \\
\hline GeneJET RNA Purification Kit & Thermo Fisher Scientific & Cat\#K0731 \\
\hline \multicolumn{3}{|l|}{ Oligonucleotides } \\
\hline qPCR Primers & IDT & Methods S1 for primer sequences \\
\hline \multicolumn{3}{|l|}{ Software and Algorithms } \\
\hline$R(v 3.6 .0)$ & http://www.R-project.org/ & $\mathrm{N} / \mathrm{A}$ \\
\hline \multicolumn{3}{|l|}{ DESeq2 package (v1.22.2) } \\
\hline SAS Software & & $\mathrm{N} / \mathrm{A}$ \\
\hline Zeiss Zen Software & $\begin{array}{l}\text { https://www.zeiss.com/mi- } \\
\text { croscopy/us/products/micro- } \\
\underline{\text { scope-software/zen-lite.html }}\end{array}$ & $\mathrm{N} / \mathrm{A}$ \\
\hline
\end{tabular}

\section{Culture of hESCs and hiPSCs}

$342 \mathrm{H} 1$ and H9 hESCs and SBli006-A hiPSCs were cultured on plates coated with vitronectin $(5 \mu \mathrm{g} / \mathrm{ml})$

343 at room temperature for at least one hour. Cells were cultured in $2 \mathrm{ml}$ of TeSR-E8 medium at

$34437^{\circ} \mathrm{C}$ in $5 \% \mathrm{CO}_{2}$ in 6 -well plates and culture medium was replaced every day. When cells reached

345 confluency, they were passaged using ReLeSR according to the manufacturer's protocol, at a

$346 \quad 1: 10$ split ratio. 


\section{Differentiation of hESCs (6 days protocol)}

348 The day after passaging, differentiation was initiated in $\mathrm{H} 1$ or $\mathrm{H} 9 \mathrm{hESC}$ by treatment with S1P $349(10 \mu \mathrm{M})$, SB431542 $(25 \mu \mathrm{M})$ and BMP4 $(20 \mathrm{ng} / \mathrm{ml})$ in TeSR-E7 for 6 days. In some experiments, 350 the S1PR agonists CYM5442 hydrochloride (10 nM), CYM5520 (5 $\mu \mathrm{M})$, CYM5541 (2 $\mu \mathrm{M})$, or the 351 Rho/ROCK inhibitor Y-27632 $(5 \mu \mathrm{M})$, or doxycycline $(2 \mu \mathrm{M})$, and/or puromycin $(1.5 \mu \mathrm{g} / \mathrm{mL})$ was 352 added during the differentiation process. The medium was replaced every day. At day 6 of treat353 ment, cells were dissociated with TrypLE for $5 \mathrm{~min}$ at $37^{\circ} \mathrm{C}$. For differentiation to EVTs, cells were 354 seeded in a 6 -well plate pre-coated with $5 \mu \mathrm{g} / \mathrm{ml}$ of vitronectin at a density of $7 \times 10^{4}$ cells per well 355 and cultured in $2 \mathrm{ml}$ of TeSR-E8 medium supplemented with SB431542 (25 $\mu \mathrm{M})$ and EGF (2.5 $\mathrm{ng} / \mathrm{ml}$ ). Medium was replaced every other day and analyzed at day 12 of total treatment. For

357 differentiation to STB, cells were seeded in a 6 -well plate pre-coated with $5 \mu \mathrm{g} / \mathrm{ml}$ of vitronectin at a density of $4 \times 10^{4}$ cells per well and cultured in $2 \mathrm{ml}$ of TeSR-E6 supplemented with Activin A (20 $\mathrm{ng} / \mathrm{ml})$ and EGF (50 ng/ml). Medium was replaced every other day and analyzed at day 14 of total treatment.

To investigate the role of YAP signaling in TB formation from hESCs, we used an hESC cell line (H9) that expresses an inducible shRNA against YAP (H9-YAP-ishRNA) (Hsiao et al., 2016). This cell line along with a scrambled shRNA control were a kind gift from Dr. Sean Palecek (University of Wisconsin). shRNA expression was induced with doxycycline under constant exposure to puromycin as the selection marker.

\section{Differentiation of hESCs to hTESCs and hTSCs}

367 The day after passaging, hESCs were differentiated by treatment with CYM5541 (2 $\mu \mathrm{M})$, SB431542 (25 $\mu \mathrm{M})$, BMP4 (20 ng/ml) in TeSR-E7 for 2 and 3 days for H1 and H9 hESCs, respectively. The medium was replaced every day. After 2 or 3 days of treatment, cells were dissociated with TrypLE for 5 minutes at $37^{\circ} \mathrm{C}$. For propagation of hTESCs, all cells were seeded in a 6 -well

371 plate pre-coated with $3 \mu \mathrm{g} / \mathrm{ml}$ of vitronectin and $1 \mu \mathrm{g} / \mathrm{ml}$ of Laminin 521 at a density of $\sim 5 \times 10^{4}$ 
372 cells per well and cultured in $2 \mathrm{ml}$ of TM4 medium [TeSR-E6 medium supplemented with

373 CYM5541 (2 $\mu \mathrm{M})$, A 83-01 (0.5 $\mu \mathrm{M})$, FGF10 (25ng/ml) and CHIR99021 (2 $\mu \mathrm{M})]$. For establish-

374 ment of hTSCs, all cells were seeded in a 6 -well plate pre-coated with $3 \mu \mathrm{g} / \mathrm{ml}$ of vitronectin and

$3751 \mu \mathrm{g} / \mathrm{ml}$ of Laminin 521 at a density of $\sim 5 \times 10^{4}$ cells per well and cultured in $2 \mathrm{ml}$ of TSCM devel-

376 oped by Okae et al. (2018) [DMEM/F12 supplemented with $0.1 \mathrm{mM} 2$-mercaptoethanol, $0.2 \%$

377 FBS, 0.5\% Penicillin-Streptomycin, 0.3\% BSA, 1\% ITS-X supplement, $1.5 \mu \mathrm{g} / \mathrm{ml}$ L-ascorbic acid,

$37850 \mathrm{ng} / \mathrm{ml}$ EGF, $2 \mu \mathrm{M}$ CHIR99021, $0.5 \mu \mathrm{M}$ A83-01, $1 \mu \mathrm{M}$ SB431542, $0.8 \mathrm{mM}$ VPA and $5 \mu \mathrm{M}$

379 Y27632]. HTESCs were directly passaged into TSCM for formation of hTSCs; complete transition

380 took $~ 5$ passages. Alternatively, hESC after 2 or 3 days of differentiation were directly passaged

381 into TSCM.

\section{Culture of hTESCs and hTSCs}

383 HTESCs and hTSCs were cultured in TM4 and TSCM, respectively, in $2 \mathrm{ml}$ of culture medium at $38437 \mathrm{C}$ in $5 \% \mathrm{CO}_{2}$. Culture medium was replaced every 2 days. When hTESCs or hTSCs reached $38570-90 \%$ confluence, they were dissociated with TrypLE at $37^{\circ} \mathrm{C}$ for $5-10$ minutes and passaged 386 to a new 6-well plate pre-coated with $3 \mu \mathrm{g} / \mathrm{ml}$ of vitronectin and $1 \mu \mathrm{g} / \mathrm{ml}$ of Laminin 521 at a 1:3387 1:4 split ratio for hTESCs and 1:4-1:6 split ratio for hTSCs. hTESCs grown in TM4 medium were 388 supplemented with Y-27632 upon passage to aid in single cell attachment. Cells were routinely 389 passaged approximately every 4-6 days. hTESCs and hTSCs at passages 5+ were used for 390 analysis, with the exception of one replicate of $\mathrm{H} 1$-derived hTESCs used in RNA-sequencing 391 analysis where cells at passage 2 in TM4 were used. hTSCs. 


\section{Differentiation of hTESCs and hTSCs}

hTSCs were grown to $\sim 80-90 \%$ confluence in TSCM and dissociated with TrypLE for 10 min at $37^{\circ} \mathrm{C}$. For differentiation to EVTs and STB, slightly modified versions of protocols developed by Okae et al. (2018) were used. For differentiation to EVTs, hTSCs were seeded in 6-well plates pre-coated with $3 \mu \mathrm{g} / \mathrm{ml}$ vitronectin and $1 \mu \mathrm{g} / \mathrm{ml}$ of Laminin 521 at a density of $1.25 \times 10^{5}$ cells per well and cultured in $2 \mathrm{~mL}$ of EVT medium (DMEM/F12 supplemented with $0.1 \mathrm{mM} 2$-mercaptoethanol, 0.5\% Penicillin-Streptomycin, 0.3\% BSA, 1\% ITS-X supplement, $100 \mathrm{ng} / \mathrm{ml}$ NRG1, $7.5 \mu \mathrm{M}$ A83-01, $2.5 \mu \mathrm{M} Y 27632$, and 4\% KSR). Matrigel was added to a final media concentration of $2 \%$ after suspending the cells in EVT medium. At day 3, the medium was replaced with the EVT medium without NRG1, and Matrigel was added to a final concentration of $0.5 \%$. At day 6 , cells were dissociated with TrypLE for $15 \mathrm{~min}$ at $37^{\circ} \mathrm{C}$ and passaged to a new vitronectin/laminincoated 6-well plates at a 1:2 split ratio. The cells were suspended in the EVT medium without

411 lin-Streptomycin, 0.3\% BSA, 1\% ITS-X supplement, $2.5 \mu \mathrm{M}$ Y27632, $2 \mu \mathrm{M}$ forskolin, and 4\% KSR.

412 The medium was replaced at day 3 , and cells were analyzed at day 6.

RNA Isolation, cDNA synthesis and Quantitative PCR.

414 RNA was isolated using Trizol ${ }^{\mathrm{TM}}$ reagent using the manufacturer's protocol. For cDNA synthesis, 415 the RNA pellet was dissolved in diethyl pyrocarbonate (DEPC)-treated water. The RNA was pu416 rified using Baseline-ZERO DNase buffer and Baseline-ZERO DNase enzyme and incubating at $41737^{\circ} \mathrm{C}$ for $30 \mathrm{~min}$. The purification was stopped with Baseline-ZERO DNase stop solution and 418 heated at $65^{\circ} \mathrm{C}$ for $10 \mathrm{~min}$. cDNA was synthesized using 18-mer Oligo-dT and dNTP mix and 419 heated to $65^{\circ} \mathrm{C}$ for $5 \mathrm{~min}$ and quickly chilled on ice. First strand buffer and DTT was added and 
420 incubated at $42^{\circ} \mathrm{C}$ for 2 min then superscript II RT enzyme was added and incubated at $42^{\circ} \mathrm{C}$ for

$42150 \mathrm{~min}$. The enzyme was inactivated at $70^{\circ} \mathrm{C}$ for $15 \mathrm{~min}$. The cDNA was stored at $-20^{\circ} \mathrm{C}$ until

422 further used. The Quantitative PCR (qPCR) reaction was carried out using SYBR Green Super-

423 mix in a C1000 Touch Thermal Cycler CFX384 Real-Time System (Rio-Rad). The primers used

424 for qPCR analysis are listed in Methods S1. ANOVA analysis of gene expression data was

425 carried out with SAS software using the $\Delta \Delta \mathrm{Ct}$ method to determine gene expression changes

426 (Livak and Schmittgen, 2001). QPCR analysis was carryout out using three biological replicates

427 for $\mathrm{H} 9$ and $\mathrm{H} 1 \mathrm{hESCs}$ as specified in the figure panels.

Immunofluorescence analysis

429 For immunofluorescence analysis, cells were grown on glass-bottom culture dishes coated with

$4303 \mu \mathrm{g} / \mathrm{ml}$ vitronectin and $1 \mu \mathrm{g} / \mathrm{ml}$ of Laminin 521 . Cells were fixed either using $4 \%$ paraformalde-

431 hyde in PBS for 10 min, permeabilized with $0.5 \%$ Triton $\mathrm{X}-100$ for 5 min and blocked in 3\%

432 BSA/PBS with $0.1 \%$ human IgG and $0.3 \%$ Triton X-100 for $1 \mathrm{hr}$. Cells were then incubated over-

433 night with the primary antibody diluted in blocking buffer. The following primary antibodies were

434 used: anti-KRT7 (SCB, 1:50), anti-KRT7 (CST, 1:500), rabbit anti-hCG (1:100), mouse anti-hCG

435 (1:100), anti-YAP (1:200), anti-TFAP2C (1:400), anti-P63 (1:600), anti-GATA3 (1:500), anti-

436 TEAD4 (1:250), anti-CDX2 (1:300), anti-VE-Cadherin (1:400), anti-HLA-G (1:300), anti-syncytin

437 (1:50). Corresponding isotype controls (rabbit polyclonal IgG, rabbit XP IgG, mouse lgG1, and

438 mouse IgG2a) were used at primary antibody concentrations. Alexa Fluor 488- or Alexa Fluor

439 647-conjugated secondary antibodies were used as secondary antibodies. Nuclei were stained

440 with DAPI and all samples were imaged using a Zeiss LSM 710 or 880 laser scanning confocal

441 microscope (Carl Zeiss, Germany). 


\section{Confocal image analysis}

443 Image analysis was conducted using an image processing algorithm created in MATLAB. First, 444 the DAPI stain was isolated, binarized, and processed to accurately represent the number of cells 445 in each image. The primary-antibody stain of interest was isolated and processed in the same 446 manner. Only primary-antibody pixels that overlap DAPI pixels were considered for analysis, and 447 the average intensities of those pixels were measured and correlated to the nearest nuclei. This was performed for one control image and multiple experimental images. Each cell in the experimental images was considered positively stained if the average intensity of that cell was greater than the average intensity of all of the cells in the control image. Statistical analysis was done using a two-tailed t-test evaluating percent positive cells from different treatment periods.

\section{RNA sequencing analysis using next generation sequencing}

453 Total RNA was extracted with Trizol ${ }^{\mathrm{TM}}$ reagent using manufacturer's protocol. RNA was purified 454 using GeneJET RNA Purification Kit using manufacturer's protocol. Isolated RNA samples were 455 then used to evaluate genome-wide mRNA expression profiles using next generation RNA-se456 quencing, conducted at GENEWIZ, LLC. (South Plainfield, NJ, USA). RNA samples received at 457 GENEWIZ were quantified using Qubit 2.0 Fluorometer (Life Technologies, Carlsbad, CA, USA) and RNA integrity was checked using Agilent TapeStation 4200 (Agilent Technologies, Palo Alto, CA, USA).

463 First strand and second strand cDNAs were subsequently synthesized. cDNA fragments were 464 end repaired and adenylated at 3'ends, and universal adapters were ligated to cDNA fragments, 465 followed by index addition and library enrichment by limited-cycle PCR. The sequencing libraries 
466

467

468

470

471

472

473

474

475

476

477

478

479

480

481

482

483

484

485

486

487

488

489

were validated on the Agilent TapeStation (Agilent Technologies, Palo Alto, CA, USA), and quantified by using Qubit 2.0 Fluorometer (Invitrogen, Carlsbad, CA) as well as by quantitative PCR (KAPA Biosystems, Wilmington, MA, USA).

The sequencing libraries were clustered on 4 lanes of a flowcell. After clustering, the flowcell was loaded on the Illumina HiSeq 4000 instrument according to manufacturer's instructions. The samples were sequenced using a 2x150bp Paired End (PE) configuration. Image analysis and base calling were conducted by the HiSeq Control Software (HCS). Raw sequence data (.bcl files) generated from Illumina HiSeq was converted into fastq files and de-multiplexed using Illumina's bcl2fastq 2.17 software. One mismatch was allowed for index sequence identification.

After investigating the quality of the raw data, sequence reads were trimmed to remove possible adapter sequences and nucleotides with poor quality using Trimmomatic v.0.36. The trimmed reads were mapped to the Homo sapiens GRCh38 reference genome available on ENSEMBL using the STAR aligner v.2.5.2b. The STAR aligner is a splice aligner that detects splice junctions and incorporates them to help align the entire read sequences. BAM files were generated as a result of this step. Unique gene hit counts were calculated by using feature Counts from the Subread package v.1.5.2. Only unique reads that fell within exon regions were counted.

\section{Analysis of gene expression profiles}

After extraction of gene hit counts, the gene hit counts table was used for downstream differential expression analysis. Genome-wide RNA sequencing count data were processed and statistically assessed using the DESeq2 package (v1.22.2) in R Software (3.6.0) (The R Foundation, 2019). Count data were first filtered to include transcripts expressed above background, requiring the median across samples to be greater than the overall median signal intensity, as implemented in DESeq2. Count data were then normalized by median signal intensity using algorithms enabled 
within DESeq2, resulting in variance stabilized expression values (Love et al., 2014). These normalized values were used to carry out a principal component analysis (PCA) comparing data-

492 reduced global expression signatures across samples. Principal components were calculated and

493 visualized using the prcomp function in R (R-core, 2019). Heat maps were generated using

494 Partek $^{\circledR}$ Genomics Suite Software (v7.18.0723) and gene-specific plots using GraphPad Prism 495 Software (v8.2.0)), based on normalized expression values.

Statistical and gene set enrichment analysis of genes differentially expressed between hTESCs and hTSCs

Genes that showed the greatest difference in expression between the hTESCs and hTSCs were identified using an analysis of variance analysis (ANOVA) comparing the normalized expression levels between these two groups. Genes showing the greatest difference in expression between

501 the hTESCs and hTSCs were identified using the following statistical filters: (1) a false discovery 502 rate-corrected q-value $<0.05$ (Storey, 2003), and (2) a fold change in expression (ratio of average across hTESCs over hTSCs samples) $\geq \pm 1.5$. To evaluate the biological role of these genes, a gene set enrichment analysis was carried out on the genes identified as significantly differentially expressed between groups. Specifically, all Gene Ontology (GO) gene sets ( $n=9996)$ from the 


\section{Acknowledgments}

514 This work was supported by NIH grants HD092741, HD093982 and NSF grant CBET 1706118

\section{$515 \quad$ Author contributions}

516 Conceptualization: AM and BR; Investigation: AM, VK, JM; Formal Analysis: AM, VK, BR, CC,

517 JR, RF; Data curation: AM, BR, JR; Resources: ASM; Writing - original draft: AM and BR; Writing

518 - review and editing: AM, BR, VK, JR, RF; Visualization: AM, VK, CC, JR; Project Administration:

519 BR; Funding Acquisition: BR and ASM

520 Declaration of interests

521 The authors declare no competing interests.

\section{Supplemental Information}

523 Document S1.pdf (contains Supplemental Figures S1-S5, Methods S1)

524 Tables S1-S4.xlsx (contains Supplemental Tables S1-S4) 


\section{References}

Amita, M., Adachi, K., Alexenko, A.P., Sinha, S., Schust, D.J., Schulz, L.C., Roberts, R.M., and Ezashi, T. (2013). Complete and unidirectional conversion of human embryonic stem cells to trophoblast by BMP4. Proc. Natl. Acad. Sci. U. S. A. 110, E1212-21.

Benirschke, K., Baergen, R.N., and Burton, G. (Graham J.. (2012). Pathology of the human placenta [electronic resource] (Heidelberg: Springer,).

Bernardo, A.S., Faial, T., Gardner, L., Niakan, K.K., Ortmann, D., Senner, C.E., Callery, E.M., Trotter, M.W., Hemberger, M., Smith, J.C., et al. (2011). BRACHYURY and CDX2 Mediate BMP-Induced Differentiation of Human and Mouse Pluripotent Stem Cells into Embryonic and Extraembryonic Lineages. Cell Stem Cell 9, 144-155.

Bischof, P., and Irminger-Finger, I. (2005). The human cytotrophoblastic cell, a mononuclear chameleon. Int. J. Biochem. Cell Biol. 37, 1-16.

Blakeley, P., Fogarty, N.M.E., del Valle, I., Wamaitha, S.E., Hu, T.X., Elder, K., Snell, P., Christie, L., Robson, P., and Niakan, K.K. (2015). Defining the three cell lineages of the human blastocyst by single-cell RNA-seq. Development 142, 3151-3165.

Van Brocklyn, J.R., Lee, M.-J., Menzeleev, R., Olivera, A., Edsall, L., Cuvillier, O., Thomas, D.M., Coopman, P.J.P., Thangada, S., Liu, C.H., et al. (1998). Dual Actions of Sphingosine-1Phosphate: Extracellular through the $\mathrm{G}_{\mathrm{i}}$-coupled Receptor Edg-1 and Intracellular to Regulate Proliferation and Survival. J. Cell Biol. 142, 229-240.

Choi, I., Carey, T.S., Wilson, C.A., and Knott, J.G. (2012). Transcription factor AP-2y is a core regulator of tight junction biogenesis and cavity formation during mouse early embryogenesis. Development 139, 4623-4632.

Cornacchia, D., Zhang, C., Zimmer, B., Chung, S.Y., Fan, Y., Soliman, M.A., Tchieu, J., Chambers, S.M., Shah, H., Paull, D., et al. (2019). Lipid Deprivation Induces a Stable, Naive-toPrimed Intermediate State of Pluripotency in Human PSCs. Cell Stem Cell 25, 120-136.e10.

Das, P., Ezashi, T., Schulz, L.C., Westfall, S.D., Livingston, K.A., and Roberts, R.M. (2007). Effects of fgf2 and oxygen in the bmp4-driven differentiation of trophoblast from human embryonic stem cells. Stem Cell Res. 1, 61-74.

Gao, X., Nowak-Imialek, M., Chen, X., Chen, D., Herrmann, D., Ruan, D., Chen, A.C.H., Eckersley-Maslin, M.A., Ahmad, S., Lee, Y.L., et al. (2019). Establishment of porcine and human expanded potential stem cells. Nat. Cell Biol. 21, 687-699.

Hemberger, M., Udayashankar, R., Tesar, P., Moore, H., and Burton, G.J. (2010). ELF5enforced transcriptional networks define an epigenetically regulated trophoblast stem cell compartment in the human placenta. Hum. Mol. Genet. 19, 2456-2467.

Home, P., Saha, B., Ray, S., Dutta, D., Gunewardena, S., Yoo, B., Pal, A., Vivian, J.L., Larson, M., Petroff, M., et al. (2012). Altered subcellular localization of transcription factor TEAD4 regulates first mammalian cell lineage commitment. Proc. Natl. Acad. Sci. U. S. A. 109, 73627367. 
Horii, M., Li, Y., Wakeland, A.K., Pizzo, D.P., Nelson, K.K., Sabatini, K., Laurent, L.C., Liu, Y., and Parast, M.M. (2016). Human pluripotent stem cells as a model of trophoblast differentiation in both normal development and disease. Proc. Natl. Acad. Sci. U. S. A. 113, E3882-91.

Hsiao, C., Lampe, M., Nillasithanukroh, S., Han, W., Lian, X., and Palecek, S.P. (2016). Human pluripotent stem cell culture density modulates YAP signaling. Biotechnol. J. 11, 662-675.

Johnstone, E.D., Chan, G., Sibley, C.P., Davidge, S.T., Lowen, B., and Guilbert, L.J. (2005). Sphingosine-1-phosphate inhibition of placental trophoblast differentiation through a $\mathrm{G}(\mathrm{i})$ coupled receptor response. J. Lipid Res. 46, 1833-1839.

Klaren, W.D., Ring, C., Harris, M.A., Thompson, C.M., Borghoff, S., Sipes, N.S., Hsieh, J.-H., Auerbach, S.S., and Rager, J.E. (2019). Identifying Attributes That Influence In Vitro -to- In Vivo Concordance by Comparing In Vitro Tox21 Bioactivity Versus In Vivo DrugMatrix Transcriptomic Responses Across 130 Chemicals. Toxicol. Sci. 167, 157-171.

Knöfler, M., Haider, S., Saleh, L., Pollheimer, J., Gamage, T.K.J.B., and James, J. (2019). Human placenta and trophoblast development: key molecular mechanisms and model systems. Cell. Mol. Life Sci. 1-18.

Knott, J.G., and Paul, S. (2014). Transcriptional regulators of the trophoblast lineage in mammals with hemochorial placentation. Reproduction 148, R121-36.

Kono, K., Tamashiro, D.A.A., and Alarcon, V.B. (2014). Inhibition of RHO-ROCK signaling enhances ICM and suppresses TE characteristics through activation of Hippo signaling in the mouse blastocyst. Dev. Biol. 394, 142-155.

Livak, K.J., and Schmittgen, T.D. (2001). Analysis of Relative Gene Expression Data Using Real-Time Quantitative PCR and the 2- $\Delta \Delta C T$ Method. Methods 25, 402-408.

Love, M.I., Huber, W., and Anders, S. (2014). Moderated estimation of fold change and dispersion for RNA-seq data with DESeq2. Genome Biol. 15, 550.

Maceyka, M., Harikumar, K.B., Milstien, S., and Spiegel, S. (2012). Sphingosine-1-phosphate signaling and its role in disease. Trends Cell Biol. 22, 50-60.

Mendelson, K., Evans, T., and Hla, T. (2014). Sphingosine 1-phosphate signalling. Development 141, 5-9.

Mo, J.-S., Yu, F.-X., Gong, R., Brown, J.H., and Guan, K.-L. (2012). Regulation of the HippoYAP pathway by protease-activated receptors (PARs). Genes Dev. 26, 2138-2143.

Moser, G., Orendi, K., Gauster, M., Siwetz, M., Helige, C., and Huppertz, B. (2011). The art of identification of extravillous trophoblast. Placenta 32, 197-199.

Nishioka, N., Yamamoto, S., Kiyonari, H., Sato, H., Sawada, A., Ota, M., Nakao, K., and Sasaki, $\mathrm{H}$. (2008). Tead4 is required for specification of trophectoderm in pre-implantation mouse embryos. Mech. Dev. 125, 270-283.

Nishioka, N., Inoue, K., Adachi, K., Kiyonari, H., Ota, M., Ralston, A., Yabuta, N., Hirahara, S., Stephenson, R.O., Ogonuki, N., et al. (2009). The Hippo signaling pathway components Lats 
and Yap pattern Tead4 activity to distinguish mouse trophectoderm from inner cell mass. Dev. Cell 16, 398-410.

Niwa, H., Toyooka, Y., Shimosato, D., Strumpf, D., Takahashi, K., Yagi, R., and Rossant, J. (2005). Interaction between Oct3/4 and Cdx2 determines trophectoderm differentiation. Cell 123, 917-929.

Ohgushi, M., Minaguchi, M., and Sasai, Y. (2015). Rho-Signaling-Directed YAP/TAZ Activity Underlies the Long-Term Survival and Expansion of Human Embryonic Stem Cells. Cell Stem Cell 17, 448-461.

Okae, H., Toh, H., Sato, T., Hiura, H., Takahashi, S., Shirane, K., Kabayama, Y., Suyama, M., Sasaki, H., and Arima, T. (2018). Derivation of Human Trophoblast Stem Cells. Cell Stem Cell 22, 50-63.e6.

R-core (2019). prcomp function | R Documentation.

Rager, J.E., Suh, M., Chappell, G.A., Thompson, C.M., and Proctor, D.M. (2019). Review of transcriptomic responses to hexavalent chromium exposure in lung cells supports a role of epigenetic mediators in carcinogenesis. Toxicol. Lett. 305, 40-50.

Ralston, A., Cox, B.J., Nishioka, N., Sasaki, H., Chea, E., Rugg-Gunn, P., Guo, G., Robson, P., Draper, J.S., and Rossant, J. (2010). Gata3 regulates trophoblast development downstream of Tead4 and in parallel to Cdx2. Development 137, 395-403.

Roberts, R.M., Loh, K.M., Amita, M., Bernardo, A.S., Adachi, K., Alexenko, A.P., Schust, D.J., Schulz, L.C., Telugu, B.P.V.L., Ezashi, T., et al. (2014). Differentiation of trophoblast cells from human embryonic stem cells: to be or not to be? Reproduction 147, D1-12.

Roberts, R.M., Ezashi, T., Sheridan, M.A., and Yang, Y. (2018). Specification of trophoblast from embryonic stem cells exposed to BMP4†. Biol. Reprod. 99, 212-224.

Sarkar, P., Randall, S.M., Collier, T.S., Nero, A., Russell, T.A., Muddiman, D.C., and Rao, B.M. (2015). Activin/Nodal Signaling Switches the Terminal Fate of Human Embryonic Stem Cellderived Trophoblasts. J. Biol. Chem. 290, 8834-8848.

Sarkar, P., Mischler, A., Randall, S.M., Collier, T.S., Dorman, K.F., Boggess, K.A., Muddiman, D.C., and Rao, B.M. (2016). Identification of Epigenetic Factor Proteins Expressed in Human Embryonic Stem Cell-Derived Trophoblasts and in Human Placental Trophoblasts. J. Proteome Res. 15, 2433-2444.

Sheridan, M.A., Yang, Y., Jain, A., Lyons, A.S., Yang, P., Brahmasani, S.R., Dai, A., Tian, Y., Ellersieck, M.R., Tuteja, G., et al. (2019). Early onset preeclampsia in a model for human placental trophoblast. Proc. Natl. Acad. Sci. U. S. A. 116, 4336-4345.

Storey, J.D. (2003). The positive false discovery rate: a Bayesian interpretation and the q value. Ann. Stat. 31, 2013-2035.

The Broad Institute (2019). GSEA | MSigDB | MSigDB Collections.

The R Foundation (2019). R: The R Project for Statistical Computing. 
Du Toit, A. (2019). Restrictions on fetal tissue research. Nat. Rev. Microbiol. 17, 462-462.

Väremo, L., Nielsen, J., and Nookaew, I. (2013). Enriching the gene set analysis of genomewide data by incorporating directionality of gene expression and combining statistical hypotheses and methods. Nucleic Acids Res. 41, 4378-4391.

Yabe, S., Alexenko, A.P., Amita, M., Yang, Y., Schust, D.J., Sadovsky, Y., Ezashi, T., and Roberts, R.M. (2016). Comparison of syncytiotrophoblast generated from human embryonic stem cells and from term placentas. Proc. Natl. Acad. Sci. U. S. A. 113, E2598-607.

Yagi, R., Kohn, M.J., Karavanova, I., Kaneko, K.J., Vullhorst, D., DePamphilis, M.L., and Buonanno, A. (2007). Transcription factor TEAD4 specifies the trophectoderm lineage at the beginning of mammalian development. Development 134, 3827-3836.

Yu, F.-X., Zhao, B., Panupinthu, N., Jewell, J.L., Lian, I., Wang, L.H., Zhao, J., Yuan, H., Tumaneng, K., Li, H., et al. (2012). Regulation of the Hippo-YAP pathway by G-protein-coupled receptor signaling. Cell 150, 780-791. 